Physica A 160 (1989) 321-350

North-Holland, Amsterdam

\title{
PHASE-LENGTH DISTRIBUTIONS IN INTERMITTENT BAND SWITCHING
}

\author{
T. POST and H.W. CAPEL \\ Instituut voor Theoretische Fysica, Universiteit van Amsterdam, Valckenierstraat 65 , \\ 1018 XE Amsterdam, The Netherlands
}

\author{
J.P. VAN DER WEELE \\ Centrum voor Theoretische Natuurkunde, Universiteit Twente, Postbus 217, \\ 7500 AE Enschede, The Netherlands
}

Received 16 June 1989

The distribution of phase lengths $t$ for intermittent band switching is investigated. Its form is observed to deviate from a exponential function; a minimal phase length is seen to exist and the probabilities for the first few occurring phase lengths are often strongly enhanced or suppressed. These deviations are analyzed and described explicitly in terms of the parameters of a model map.

\section{Introduction}

Historically, the concept of intermittency stems from fluid dynamics [1-3], where it is used to describe the transition from a laminar to a turbulent flow in various situations, as some system parameter is varied.

Pomeau and Manneville [4] conjectured that this transition might be qualitatively understood by a new route to chaos in one-dimensional raps. The intermittent behaviour in this route occurs when for certain values of a control parameter the orbit is confined for long times to a small part of the interval, evolving in a regular fashion, to escape from time to time exhibiting short chatic bursts. In analogy with the fluid dynamics intermittency the long regular stretches were called laminar phases.

At present, other intermittency routes to chaos have been discovered in various low-dimensional maps [5-10], providing possible models for a wide range of experimental situations, concerning for instance optical bistability [11], biochemistry [12], forced pendula (and hence Josephson junctions and VCOs) [13] and diode resonators [14]. These new types of intermittent behaviour differ from the Pomeau-Monneville (PM) types [4] in that during 
the periods of confinement to a small part of the interval they also exhibit chaotic behaviour, only on a smaller scale.

This paper will treat an intermittent phenomenon that occurs when two chaotic bands merge $[5-7,9,11,12,15-17]$. Just after the merger of the two chaotic bands the orbit of the twice iterated map is found to spend relatively long periods in either of the former band regions, but at irregular times it switches from one region to the other. This phenomenon has been called intermittent hopping or switching and an exponential (geometrical) distribution of the periods $t$ (phase lengths) between subsequent switches has been observed.

However, in numerical investigations we find that the phase length $t$ cannot be arbitrarily short. That is, a minimal phase length $t_{\min }$ exists, which increases when we get nearer to the merging point. Moreover, the probability distribution fluctuates strongly for the shortest phase lengths, showing high peaks and dips.

It is the purpose of this paper to give a quantitative description of the above short-phase phenomena, using the map

$$
f(x)=1-a|x|^{z}, \quad z>1,
$$

as an example.

In the next section we present a brief overview of the various existing intermittent phenomena and their underlying mechanisms. Furthermore, we give a qualitative account of their individual scaling behaviours and we discuss the form of their probability distributions.

In section 3 we discuss the band structure of the map (1), focussing on the band merging process and the icl mpanying switching mechanism.

In section 4 we explain the existence of a minimal period $t_{\min }$ and we derive an expression for it in terms of the small parameter $\varepsilon=a-a_{1}$, where $a_{1}$ denotes the value for $a$ for which the two chaos bands merge into one.

In section 5 we derive explicit expressions for the probabilities of the shortest phase lengths in terms of $\varepsilon$ and the order $z$ of the maximum, explaining the strong short-phase fluctuations in the phase-length distributions. The results are confronted with numerically obtained phase-length distributions.

Finally, in section 6 we discuss the window structure of the map (1) close to the band merging value $a_{1}$, which inappens to be an accumulation point of windows of period $2 n+1, n=-1,2, \ldots$ [17]. These windows are closely related to the minimal phase lergths $t_{\min }=n$. Furthermore, we expect PM type 1 intermittency [4] and intermittent bursting [9] at the borders of the windows. Thus an intricate interplay of various intermittencies takes place at these specific parameter values. 


\section{An overview of intermittent phenomena}

In this section we discuss the intermittency routes to chaos for the map (1).

When the parameter $a$ is increased from 0 up to $a_{0}=2$ we observe the following phenomena, as illustrated in fig. 1 below.

For $a<(z+1)^{z-1} / z^{z}$ the orbit is attracted to the positive fixed point $X$ of the map. As $a$ is increased up to $a_{\infty}$ the orbit undergoes the well-known cascade of period-doubling bifurcations. At $a=a_{\infty}$ we have an orbit of period $2^{\infty}$, consisting of points which together form a Cantor set. (For a discussion of its dimension see e.g. ref. [18].) For $a>a_{\infty}$ the attractor is composed of a collection of chaos bands which undergoes a sequence of reverse bifurcations, or band mergings, at values $\ldots, a_{3}, a_{2}, a_{1}$. Here $a_{n}$ denotes the value of $a$ at which $2^{n}$ chaos bands merge into $2^{n-1}$ bands, in a pairwise manner. For $a$ just above $a_{n}$ the orbit of the $2^{n}$ times iterated map shows intermittent hopping or switching (IS). Beyond $a=a_{1}$ we have one large chaos band.

The whole range $a>a_{\infty}$ however is densely intersparsed with so-called windows in which the orbit is periodic. The opening of a period $n$ window is marked by a tangent bifurcation of the $n$ times iterated map $f^{n}(x)$, i.e. a stable/unstable pair of $n$-cycles is born at, say, $a=a_{\text {open }}$. For $a$ just below $a=a_{\text {open }}$ PM type 1 intermittency is observed. Inside the period $n$ window the stable $n$-cycle also undergces the cascade of period doublings and the subsequent band mergings until we have an $n$-piece attractor.

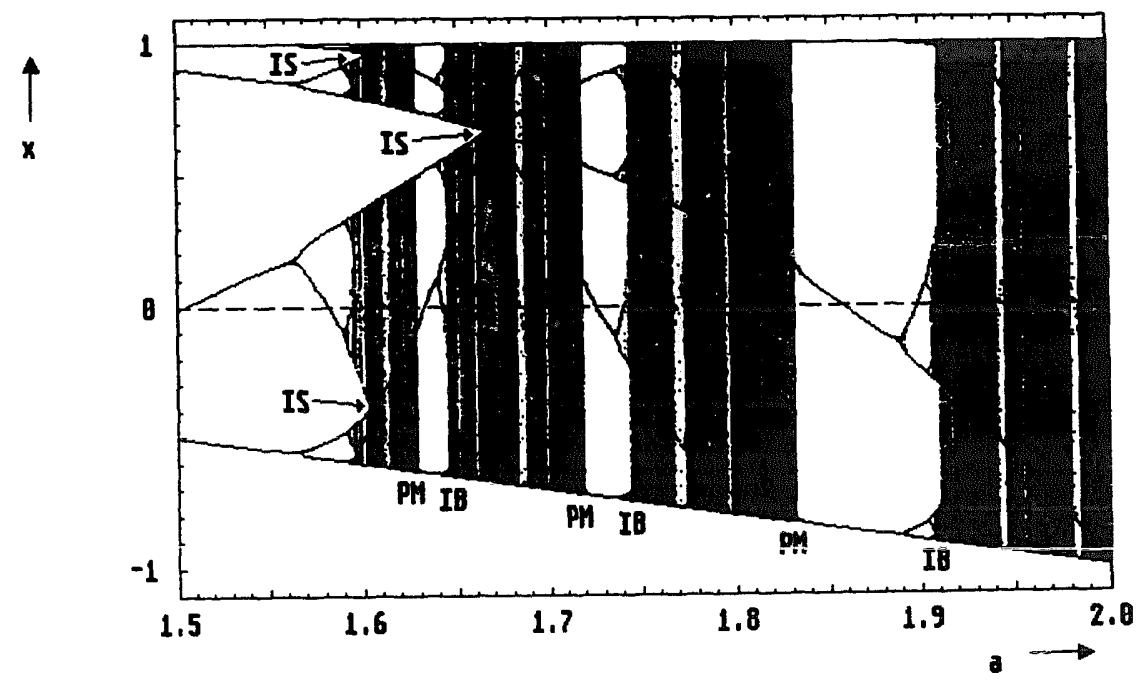

Fig. 1. The structure of the attractor for the map $f(x)=1-a x^{4}$, for $1.5<a<2$. We have indicated some parameter values where the various kinds of intermittent behaviour are to be expected. PM stands for Pomeau-Manneville type 1 intermittency, IB stands for intermittent bursting and IS for intermittent switching. 
When this $n$-piece attractor finally collides with the unstable $n$-cycle from the tangent bifurcation the window closes. For $a$ just above $a_{\text {close }}$ we then observe a phenomenon which is called intermittent bursting (IB).

We shall now briefly review the mechanisms behind these intermittencies.

\subsection{Pomeau-Manneville type 1 intermittency}

To explain the mechanism of PM type 1 we focus on the largest periodic window in the parameter space of the map (1), the 3-window, with $a_{\text {open }}=1.75$ for $z=2$.

In fig. 2 the three times iterated map is plotted for $a=1.749$, just below $a_{\text {open }}$ where the tangent bifurcation takes place. In fact, the plotted function is almost tangent to the line $x=y$ at three places. We observe that an orbit which comes close to a near-tangency gets caught in the narrow channel between the function and the line $y=x$. The evolution of the orbit inside the narrow channel is called the laminar phase. After a number of iterations the orbit gets out of the channel and is free to wander around chaotically until accidentally it comes close to a near-tangency again, and so on.

The width $d$ of the channel is proportional to

$$
\varepsilon=a_{\mathrm{open}}-\boldsymbol{a}
$$

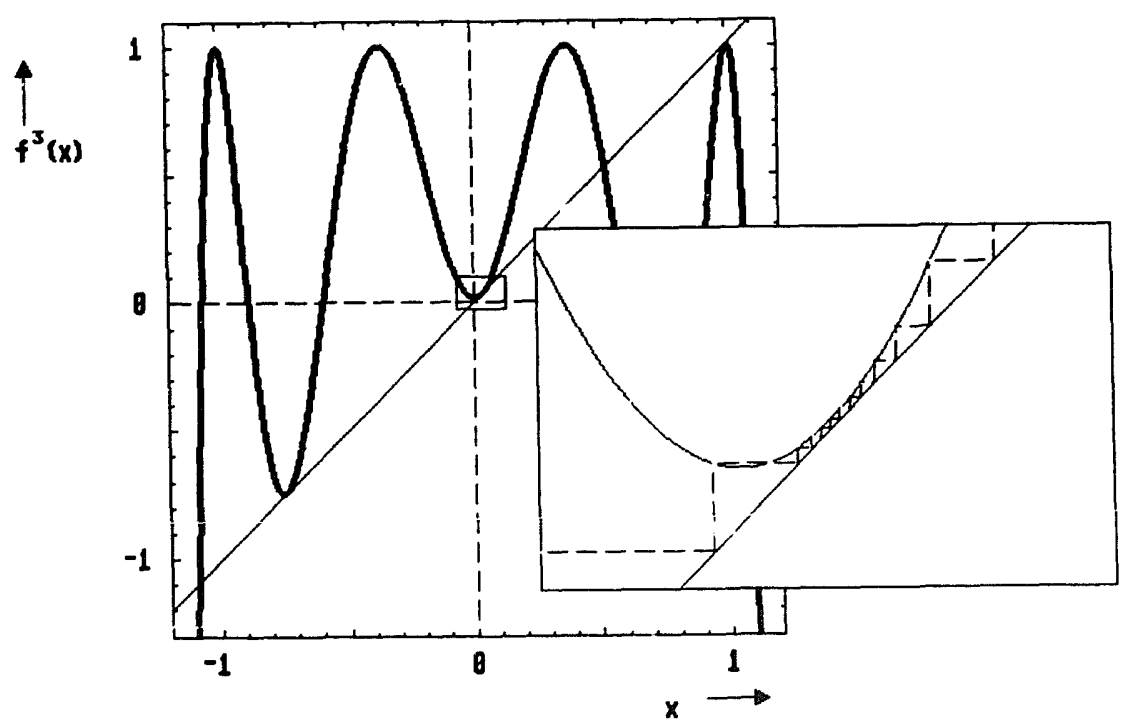

Fig. 2. The map $f^{3}(x)$ for $z=2$ at $a=1.749$, before the opening of the 3-window at $a_{\text {open }}=1.75$. The plotted function is almost tangent to the line $x=y$ at three places, as can be verified watching the blow-up of the small rectangle covering the central near-tangency. Some iterations of $f^{3}(x)$ in the channel discussed in the text are shown (dashed lines). 
Since the maximum of the map (1) is of order $z$, all extrema of the $n$ times iterated map will be of order $z$ as well, so the length $L$ of the channel is proportial to $\varepsilon^{1 / z}$. Now, the mean number of iterations $\langle t\rangle$ an orbit has to spend inside a channel will be proportional to the length $L$ of the channel divided by the average distance the orbit passes per iteration, which is proportional to the channel width $d$. Putting this together we estimate for the mean length $\langle t\rangle$ of a laminar phase:

$$
\langle t\rangle \sim \varepsilon^{1 / z} / \varepsilon=\varepsilon^{-(z-1) / z} .
$$

This relation can also be found in a rigorous manner using renormalization group arguments $[19,20]$. The success of this approach is due to the fact that the Feigenbaum-Cvitanović equation [21] can be solved exactly in this case.

\subsection{Crisis-induced intermittencies}

The mechanisms for intermittent bursting (IB) and switching (IS) are essentially the same as the mechanism for transients to infinity for $a$ just beyond $a_{0}=2[8,9]$. The event which causes the intermittent or transient phenomena to occur is a so-called crisis [6], i.e. the collision of a chaotic attractor with an unstable cycle. For values of the parameter $a$ just past the value where the crisis occurs an orbit of the map is confined to a part of the interval for a finite number of iterations, after which it escapes to

- minus infinity, in the case of transient behaviour;

- a larger, embedding part of the interval, in the case of intermittent bursting;

- another, adjoining part of the interval, in the case of intermittent switching.

We discuss the mechanism for transient phenomena first, then we generalize it to the IB and IS situations. We give a detailed treatment here, since this is a prerequisite for a full understanding of the anomalies that occur in intermittent switching.

In fig. 3 the map (1) is drawn for $z=3$ at $a=1.8$ and $a=2.1$. We have used the negative fixed point $x_{-}$and its nontrivial pre-image $f^{-1}\left(x_{-}\right)$to construct a square box, with corners $\left(x_{-}, x_{-}\right),\left(f^{-1}\left(x_{-}\right), x_{-}\right),\left(f^{-1}\left(x_{-}\right), f^{-1}\left(x_{-}\right)\right)$and $\left(x_{-}, f^{-1}\left(x_{-}\right)\right)$. We see that in fig. 3a the maximum of the mapping at $\tilde{x}_{0}=0$ is completely contained within the box. Its value $\tilde{x}_{1} \stackrel{\text { def }}{=} f\left(\tilde{x}_{0}\right)=1$ gives the upper boundary for the chaotic attractor. The lower boundary is obtained by iterating

the upper one once: $\tilde{x}_{2} \stackrel{\text { def }}{=} f\left(\tilde{x}_{1}\right)=1-a$. Furthermore, the box denotes the basin of attraction of the chaotic attractor, i.e. all orbits started within it will be drawn into the attractor. Any point outside the box is mapped on one iteration to the left of $x_{-}$, and successively to minus infinity. 

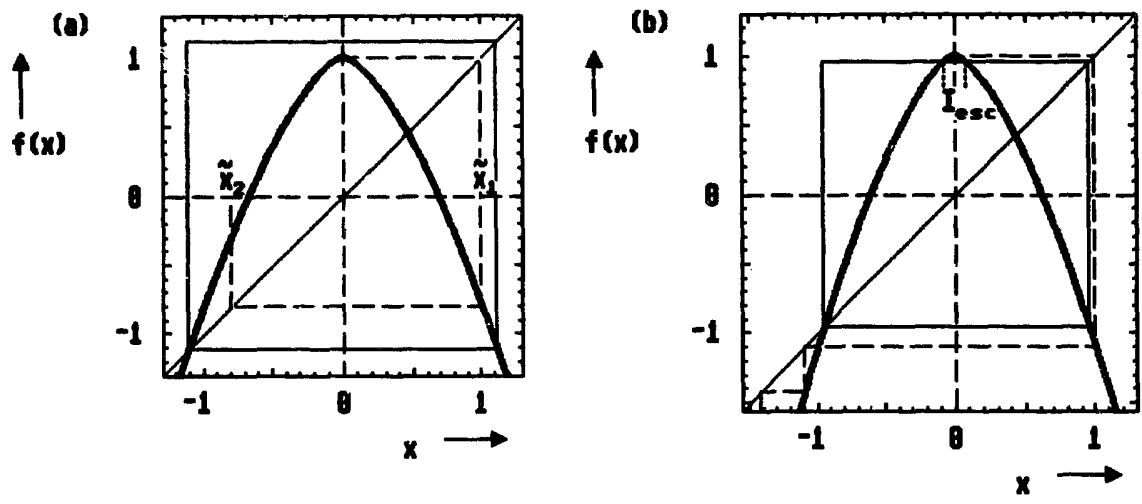

Fig. 3. The map $f(x)$ for $z=1.5$ (a) at $a=1.8$. The boundaries of the attractor are indicated by $\tilde{x}_{2}$ and $\tilde{x}_{1}$, respectively. Note that the maximum at $\tilde{x}_{0}=0$ is completely contained within the square box discussed in the text. (b) at $a=2.1$. The maximum at $\tilde{x}_{0}=0$ pierces through the square box discussed in the text. Some iterations of a typical orbit which escapes to $-\infty$ after landing in the escape region $I_{\text {esc }}$ are shown (dashed lines).

For all $a<2$ the maximum is contained within the box and hence the attractor is stable, i.e. an orbit once on it will remain there forever. At $a=2$ the maximum hits the top of the box, as can be checled easily; in this situation the left attractor boundary $\tilde{x}_{2}$ coincides with the negative fixed point $x_{-}$(so we have a crisis here), while the symmetry of the map dictates that $x_{-}=-\tilde{x}_{1}=$ -1 . Thus we have $\tilde{x}_{2}=1-a=-1$, hence $a=2$ for all $z$. For $a$ above $a_{0}=2$ the maximum pierces through the iop of the box, as illustrated in fig. $3 \mathrm{~b}$. The interval where $f(x)$ exceeds the box denotes the escape region $\mathrm{I}_{\mathrm{esc}}$. Any orbit point which falls into this interval is mapped to the right side of the box and on the next iteration to the left side, whereafter it wanders away to minus infinity.

For small $\varepsilon=a-a_{0}$ the amount $d$ that $f(x)$ exceeds the box is proportional to $\varepsilon$, so the width $L$ of the escape region is proportional to $\varepsilon^{1 / z}$. For small values of $\varepsilon$ this is also a small quantity, and orbits of $f(x)$ started inside the box will in general remain there for long times before escaping to minus infinity. The mean number of iterations $\langle t\rangle$ an orbit with a random initial condition within the box spends before escaping can thus be estimated as

$$
\langle t\rangle \sim 1 / L \sim \varepsilon^{-1 / z} .
$$

We denote the iterations an orbit spends within the box as the transient phase. We shall now discuss the distribution $P(t)$ of lengths $t$ of transient phases for orbits with random initial conditions within the box, for small $\varepsilon$. In this case we have a small escape region: $L \ll 1$, so we can assign a small probability $q=1 /\langle t\rangle$ for an orbit to land in it on each iteration. Then the probability for a transient phase of length $t$ to occur equals 


$$
P(t)=(1-q)^{t-1} q=\frac{1}{\langle t\rangle} \exp \left[(t-1) \ln \left(1-\frac{1}{\langle t\rangle}\right)\right]
$$

This is a geometrical distribution. Since for small $a$ we have $\ln (1-q)=$ $-q+\mathcal{O}\left(q^{2}\right)$ the distribution limits for large $\langle t\rangle$ to an exponential one,

$$
P(t)=\frac{1}{\langle t\rangle} \exp \left[-\frac{t}{\langle t\rangle}\right]
$$

For a discussion of this distribution see also ref. [14].

\subsection{Intermittent bursting}

In order to understand intermitticnt bursting (IB) we consider the thres tinte. iterated map $f^{3}(x)$ for $z=3$ at $a=1.86$, as depicted in fig. 4, iust before the closing of the 3-window at $a_{\text {close }}=1.869904234 \ldots$ We can now irow thee square boxes in the same manner as before using the unstable 3-cycle elements and their closest nontrivial pre-images under $f^{3}(x)$ as reference points. Each of these boxes contains a (sub)map similar to the original map $f(x)$, but on a smaller scale.

As long as the extrema of the three submaps lie inside their boxes (as in fig. 4 ), any orbit of $f^{3}(x)$ trapped in one of them will remain there forever. So,

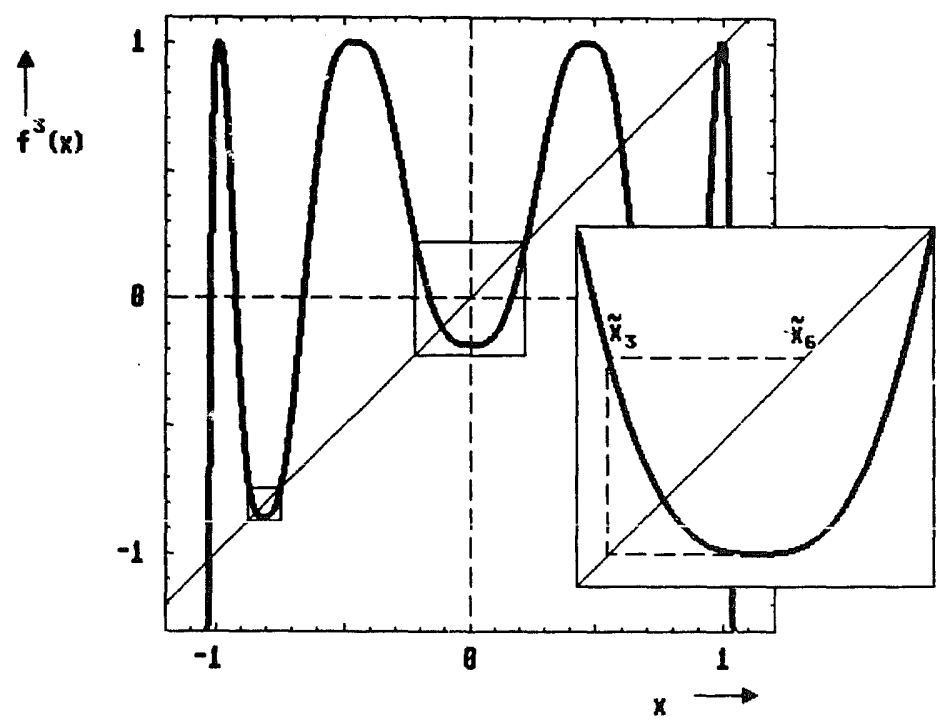

Fig. 4. The map $f^{3}(x)$ for $z=3$ at $a=1.86$, before the closing of the 3-window at $a_{\text {close }}=$ $1.869904234 \ldots$ The boundaries of the three bands are $\tilde{x}_{2}, \tilde{x}_{5}$ and $\tilde{x}_{3}, \tilde{x}_{6}$ and $\tilde{x}_{4}, \tilde{x}_{1}$, respectively (see text). In the insert a blow-up of the central box is drawn. Note that the three submaps discussed in the text are completely contained within the square boxes. The rightmost box is so small it cannot be discerned in this figure. 
considering $f(x)$, we have a three-piece chaotic attractor here. The actual chaos bands are given by the intervals $\left[\tilde{x}_{2}, \tilde{x}_{5}\right],\left[\tilde{x}_{3}, \tilde{x}_{6}\right]$ and $\left[\tilde{x}_{4}, \tilde{x}_{1}\right]$, where

$$
\tilde{x}_{n}=f^{n}(0)
$$

denotes the $n$th iterate of the maximum $\tilde{x}_{0}=0$, i.e. $\tilde{x}_{1}=1, \tilde{x}_{2}=1-a, \tilde{x}_{3}=$ $1-a|1-a|^{z}$, and so on.

At $a=a_{\text {close }}$ the extrema of the submaps hit their boxes simultaneously, and at this moment we have $x_{-, i}=\tilde{x}_{4+i}, i=0,1,2$, where $x_{-, i}$ denotes the $i$ th element of the unstable 3-cycle, so here the attractor collides with the unstable cycle.

For $a>a_{\text {close }}$ we have a situation similar to the one in fig. $3 \mathrm{~b}$. The three extrema pierce through their boxes and consequently an orbit can escape from its box. For small values of $\varepsilon=a-a_{\text {close }}$ the escape regions, which are given by the intervals where $f^{3}(x)$ exceeds the boxes, are quite smali, and orbits of $f^{3}(x)$ will in general remain for long times in one of the former band regions. When the orbit escapes it wanders around chaotically within the overall attractor boundaries $\tilde{x}_{2}$ and $\tilde{x}_{1}$, until it gets trapped again in one of the bexes, and so on. A iypical time series of $f^{3}(x)$ is shown in fig. 5 .

The average time $\langle t\rangle$ an orbit spends in the former band regions is again proportional to $\varepsilon^{-1 / z}$, cf. eq. (4). Since the boxes are relatively small as compared to the whole interval $\left[\tilde{x}_{2}, \tilde{x}_{1}\right]$ and since the motion outside the boxes is chaotic, we may assume that the points where the orbit re-enters the boxes

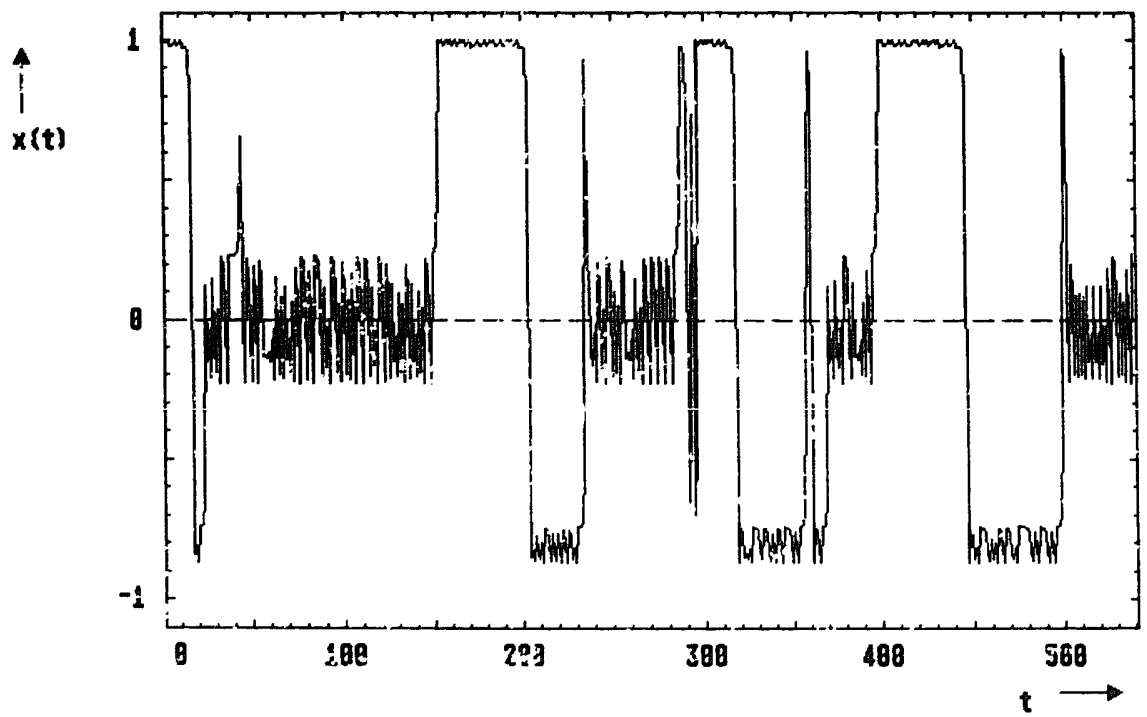

Fig. 5. A time series of the map $f^{3}(x), f(x)=1-a|x|^{3}$, for $a=1.87$. just afier the closing of the 3-window at $a_{\text {close }}=3.869904234$.. Sticcessive iterates are joined by straight lines for ciarity. 
are more or less randomly distributed. This means the distribution of the lengths of the confinement phrses is given by (6).

\subsection{Intermittent switching}

Finally, we discuss the phenomenon of intermittent switching (IS). In fig. 6 the twice iterated map $f^{2}(x)$ is depicted for $z=2, a=1.51$, just before the last band merging at $a_{1}=1.5436890 \ldots$ Two boxes containing a submap can be consiructed now, taking as reference points the positive fixed point $X$ and its pre-images under $f^{2}(x)$ at the left and the right of $X$ :

$$
X_{\mathrm{L}}=-X, \quad X_{\mathrm{R}}=\left(\frac{1+X}{a}\right)^{1 / 2} .
$$

For $a<a_{1}$, before the band merging, the extrema of the submaps lie inside their boxes, so an orbit of $f^{2}(x)$ started in either of them remains there forever. The actual chaos bands are given by the intervals $\left[\tilde{x}_{2}, \tilde{x}_{4}\right]$ and $\left[\tilde{x}_{3}, \tilde{x}_{1}\right]$. At $a=a_{1}$ the extrema hit their boxes simultaneously, and the band boundaries $\tilde{x}_{4}$ and $\tilde{x}_{3}$ touch each other (at $X$ ). For $a$ just above $a_{1}$ the points $\tilde{x}_{4}$ and $\tilde{x}_{3}$ have crossed each other, and orbits of $f^{2}(x)$ can escape from their boxes, again with a small probability proportional to $\varepsilon^{1 / 2}$, with $\varepsilon=a-a_{1}$ here. In doing so, the orbit is first transferred to the left of $X_{\mathrm{L}}$, respectively, the right of $X_{\mathrm{R}}$, and on the next iteration of $f^{2}(x)$ it enters the inierval $\left[X, \tilde{x}_{4}\right]$, respectively, $\left[\tilde{x}_{3}, X\right]$ of the other box. So, for small $\varepsilon$ an orb:t of the twice iterated map is found to

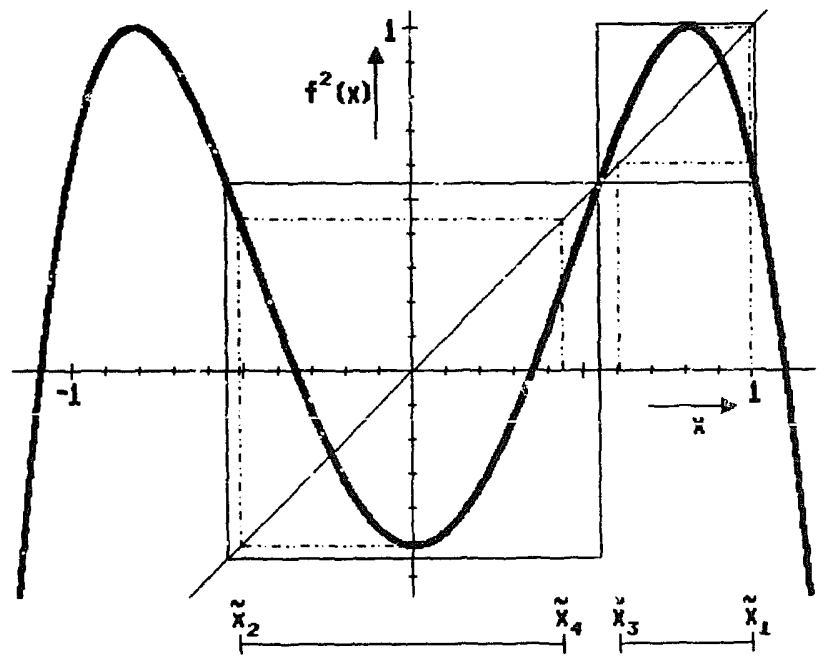

Fig. 6. The map $f^{2}(x)$ for $z=2$ at $a=1.51$, before the band merging at $a=a_{1}=1.5436890$. The bounuaries of the two bands are indicated by $\tilde{x}_{2}, \tilde{x}_{4}$ and $\tilde{x}_{3}, \tilde{x}_{1}$, respectively. Note that the two submaps discussed in the text are completely contained within the square boxes. 
spend relatively long periods in either of the former band regions, but at irregular times it switches from one region to the other. A typical time series at $a=1.545\left(\varepsilon=1.31110^{-3}\right)$ is shown in fig. 7 .

The average duration of the phases scales again with $\varepsilon^{-1 / z}$ in the limit $\varepsilon \rightarrow 0$, but we must be careful in making assumptions about the distribution $P(t)$ of phase lengths $t$, since the entrance points in the boxes are not randomly distributed over the boxes, but are confined to the small intervals $\left[\tilde{x}_{3}, X\right]$ for the left box and $\left[X, \tilde{x}_{4}\right]$ for the right box. Hence we expect the distribution (6) to hold only for the longer phase lengths. We shall come back to this in the following sections.

\section{Band structure and switching process}

As already mentioned before, the switching phenomenon occurs for all $a$-values just above all band merging values $a_{n}$. In this section we shed some light on how the band structure of the map (1) comes about and we highlight the switching process more in detail.

\subsection{Band structure of the attractor}

In the previous section we studied the last band merging at $a=a_{1}$ by looking at the twice iterated map $f^{2}(x)$. We continue this line of thought by considering the four times iterated map just before the one but last band merging at $a=a_{2}$.

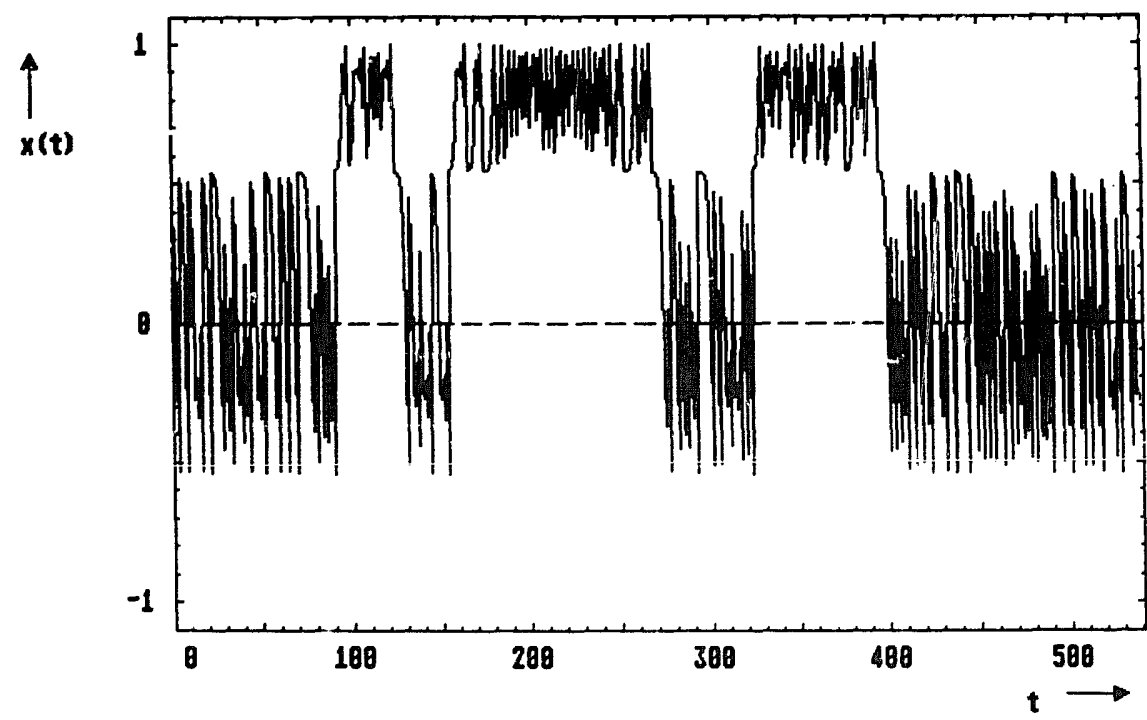

Fig. 7. A time series of the map $f^{2}(x), f(x)=1-a x^{2}$, for $a=1.545$, just after the band merging at $a_{1}=1.5436890$. Successive iterates are joined by straight lines for clarity. 
A plot of this map is given in fig. 8 for $z=1.1$ at $a=1.2$. For $z=1.1$ we have $a_{2}=1.2343723 \ldots$

Now we construct four boxes taking as reference points the 2-cycle and their respective pre-images under $f^{4}(x)$ at their immediate left and right. These four boxes all contain submaps whose extrema touch the boxes at the band merging value $a_{2}$. Just above $a=a_{2}$ orbits of $f^{4}(x)$ show intermittent switching between the two boxes of the pair in which they were started. In the figure we have also drawn the boxes with as reference points the positive 1-cycle $X$ and its pre-images under $f^{2}(x)$, cf. eq. (8). In the depicted situation $\left(a<u_{2}\right)$ the attractor bands are bounded by the extremal values of the submaps and their images under $f^{4}(x)$. They are the intervals $\left[\tilde{x}_{2}, \tilde{x}_{6}\right],\left[\tilde{x}_{8}, \tilde{x}_{4}\right],\left[\tilde{x}_{3}, \tilde{x}_{7}\right]$ and $\left[\tilde{x}_{5}, \tilde{x}_{1}\right]$.

It can easily be proved that all extremal values of an $n$-times iterated unimodal map $f(x)$, i.e. $f^{n}(x)$, are given by the values $\tilde{x}_{1}, \tilde{x}_{2}, \ldots, \tilde{x}_{n}$, where $\tilde{x}_{i}$ denotes the $i$ th iterate of the critical point $\tilde{x}_{0}$ of $f(x)$, cf. eq. (7). The ordering of the iterates $\tilde{x}_{i}, i=1,2, \ldots, 2 n$, follows from the theory of Metropolis, Stein and Stein (MSS) [22]. We elaborate on this in appendix A.

At $a=a_{2}$ we have $\tilde{x}_{5}=\tilde{x}_{7}$ and hence $\tilde{x}_{6}=\tilde{x}_{8}$, which illustrates the fact that the merging points of the attractor bands are given by the 2-cycle, just like at $a=a_{1}$ we have $\tilde{x}_{3}=\tilde{x}_{4}$, where the merging point is the (positive) 1-cycle. In genera! at $a=a_{n}$ we have $\tilde{x}_{2^{n}+1}=\tilde{x}_{2^{n}+1+2^{n-1}}$. This is the condition one normally uses to determine the band merging values $a_{n}$.

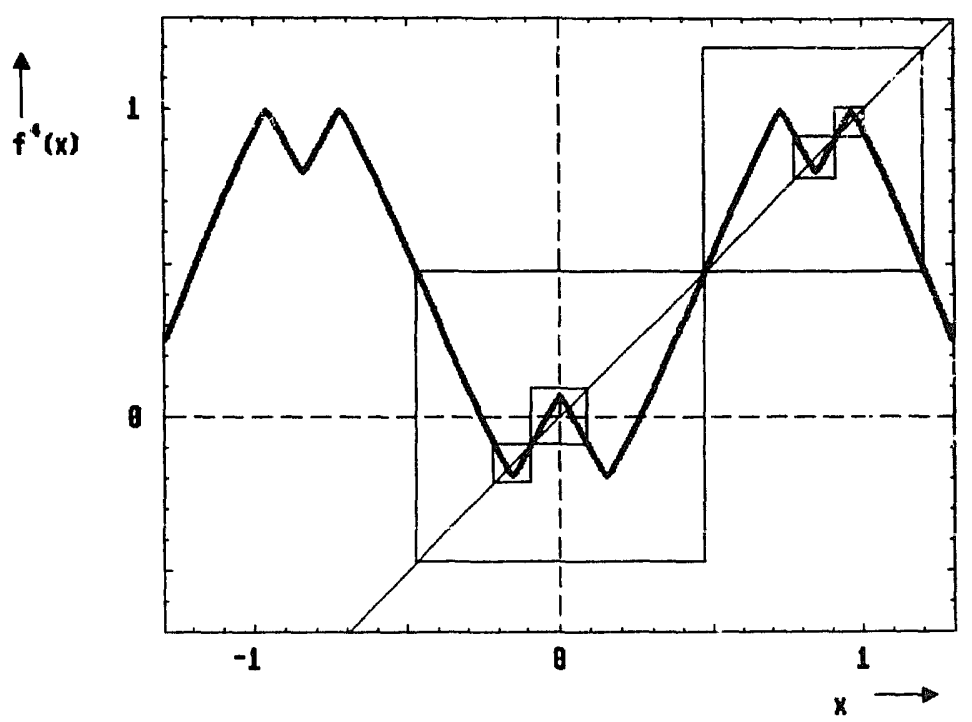

Fig. 8. The map $f^{4}(x)$ for $z=1.1$ at $a=1.2$, before the band merging at $a=a_{2}=1.2343723 \ldots$ The boundaries of the four bands are $\tilde{x}_{2}, \tilde{x}_{6}$ and $\tilde{x}_{8}, \tilde{x}_{4}, \tilde{x}_{3}, \tilde{x}_{7}$ and $\tilde{x}_{5}, \tilde{x}_{1}$, respectively. Note that the four submaps discussed in the text are completely contained within their boxes. Also drawn are the boxes corresponding to the submaps of $f^{2}(x)$. 
Some band merging values for the map (1) are given in table $I$, for various values of $z$. They converge geometrically to $a_{\infty}(z)$, the accumulation point for period doubling, i.e. $\delta_{n}=\left(a_{n}-a_{n-1}\right) /\left(a_{n+1}-a_{n}\right), \delta_{n} \rightarrow \delta(z)$, with $\delta(z)$ being equal to the well-known Feigenbaum constant for period doubling $[2,18]$. Moreover, the widths of the bands at successive $a_{n}$ 's scale according to the scaling function $1 / \sigma(z)$ of Feigenbaum [21,18]. In the table we also give the scaling of the quantity $\tilde{x}_{2^{n+1}}-\tilde{x}_{2^{n}}$, whose absolute value is the width of the largest band (the one that contains $\tilde{x}=0$ ). This quantity scales rather nicely with the factor $\alpha_{n}, \alpha_{n} \rightarrow \alpha(z)$.

Table I

Band merging values and the scaling approximants $\delta_{n}$ and $\alpha_{n}$ as discussed in the text for some values of $z$.

\begin{tabular}{|c|c|c|c|}
\hline$n$ & $a_{n}$ & $\delta_{n}$ & $\alpha_{n}$ \\
\hline \multicolumn{4}{|c|}{$z=1.1$} \\
\hline 1 & 1.4317116724407 & & \\
\hline 2 & 1.2343722800140 & 2.815553 & --6.088603 \\
\hline 3 & 1.1642832538048 & 2.800400 & -7.180582 \\
\hline 4 & 1.1392550372393 & 2.815698 & -7.669212 \\
\hline 5 & 1.1303662234870 & 2.824695 & -7.858585 \\
\hline 6 & 1.1272194006324 & 2.828441 & -7.927802 \\
\hline 7 & 1.1261068362159 & 2.829841 & -7.952554 \\
\hline 8 & 1.1257136818428 & & \\
\hline \multicolumn{4}{|c|}{$z=2$} \\
\hline 1 & 1.5436890126921 & & \\
\hline 2 & 1.4303576324513 & 4.937646 & -2.464982 \\
\hline 3 & 1.4074051181647 & 4.671847 & -2.496139 \\
\hline 4 & 1.4024921763586 & 4.675955 & -2.501243 \\
\hline 5 & 1.4014414942536 & 4.669907 & -2.502571 \\
\hline 6 & 1.4012165043094 & 4.669443 & -2.502832 \\
\hline 7 & 1.4011683208393 & 4.669242 & -2.502892 \\
\hline 8 & 1.4011580015052 & 4.669212 & -2.502904 \\
\hline 9 & 1.4011557914246 & 4.669204 & -2.502907 \\
\hline 10 & 1.4011553180932 & & \\
\hline \multicolumn{4}{|c|}{$z=3$} \\
\hline 1 & 1.6180339887499 & & \\
\hline 2 & 1.5366213864468 & 6.616745 & -1.917654 \\
\hline 3 & 1.5243173613342 & 6.035730 & -1.928489 \\
\hline 4 & 1.5222788300306 & 6.098311 & -1.927491 \\
\hline 5 & 1.5219445520154 & 6.082926 & -1.927745 \\
\hline 6 & 1.5218895985208 & 6.085109 & -1.927690 \\
\hline 7 & 1.5218805677057 & 6.084615 & -1.927694 \\
\hline 8 & 1.5218790835010 & 6.084703 & -1.927691 \\
\hline 9 & 1.5218788395771 & 6.084686 & -1.927691 \\
\hline 10 & 1.5218787994889 & & \\
\hline
\end{tabular}


These facts are not too surprising, since to derive the relevant scaling laws one uses the same renormalization scheme, with the same scaling operation $T \circ g(x)=\alpha g(g(x / \alpha))$ and the same accumulation point $a_{x}$.

To appreciate the fact that the attractor has a (multi)fractal structure at $a=a_{\infty}$, one gains a more direct insight in this siructure when one approaches $a_{\infty}$ from above instead of from below, as is usually done. Considering the attractor at successive values $a=a_{n}$ we first see one interval at $a=a_{1}$. At $a=a_{2}$ the middle part is taken out in such a way that the remaining parts have approximately $1 / \alpha$ resp. $1 /|\alpha|^{z}$ times the size of the original interval. At $a=a_{3}$ this process repeats for each of the remaining intervals, and so on until at $a=a_{\infty}$ we have a Cantor set.

\subsection{A symbolic description of the switching process}

We shall now have a closer look at the switching process occurring after a band merging. For this purpose we consider the original map $f(x)$ for $z=1.5$ at $a=1.6$, just after the last band merging at $a_{1}=1.48963190 \ldots$, as depicted in fig. 9.

Before the band merging an orbit always alternates between the regions 1 and $r$ at the left, respectively, the right side of the positive fixed point $X$. After the band merging we have the situation as in fig. 9. We have also drawn the two square boxes discussed in the previous section with the reference points $X$ and its pre-images $X_{\mathrm{L}}$ and $X_{\mathrm{R}}$ under $f^{2}(x)$, cf. (8). Also the attractor

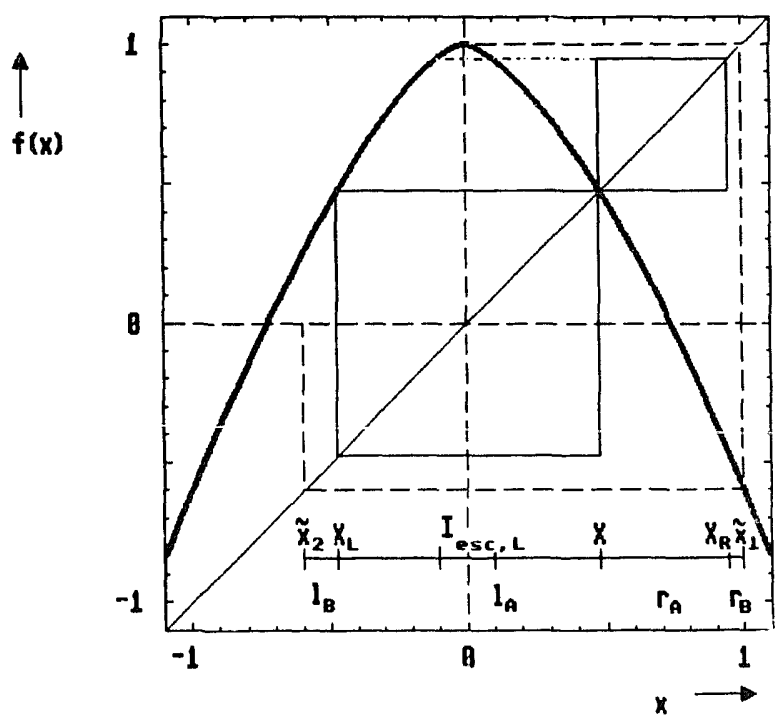

Fig. 9. The map $f(x)$ for $z=1.5$, at $a=1.6$, after the band merging. The boundaries of the attractur are $\tilde{x}_{1}=1$ and $\tilde{x}_{2}=1-a$. Also drawn are the two square boxes discussed in the text with the reference points $X$ and its pre-images $X_{\mathrm{L}}$ and $X_{\mathrm{p}}$ ur.ier $f^{2}(x)$. 
boundaries $\tilde{x}_{1}$ and $\tilde{x}_{2}$ are indicated. Now the left part $1=\left[\tilde{x}_{2}, X\right]$ and the right part $\mathrm{r}=\left[X, \tilde{x}_{1}\right]$ of the attractor are divided into the regions $1_{\mathrm{A}}=\left[X_{\mathrm{L}}, X\right]$, $\mathbf{r}_{\mathrm{A}}=\left[X, X_{\mathrm{R}}\right]$, which are covered by the left, respectively, the right box, and the regions $1_{B}=\left[\tilde{x}, X_{L}\right], r_{B}=\left[X_{R}, \tilde{x}_{1}\right]$, which are not.

We observe, considering the figure, that a point in region $1_{B}$ is always mapped into $1_{A}$, a point in $r_{B}$ always into $1_{B}$, a point in $r_{A}$ always into $1_{A}$, but a point in $1_{A}$ can be mapped into both $r_{A}$ and $r_{B}$, depending on whether it lies in the subinterval denoted by $I_{e s c, L}$ or not. From these observations we deduce the following alternation rules:

$$
\begin{aligned}
r & \rightarrow 1, \\
1 & \rightarrow \mathbf{r} \text { or } 1, \\
11 & \rightarrow \mathrm{r} .
\end{aligned}
$$

Since for small values of $\varepsilon=a-a_{1}$ the interval $I_{e s c, L}$ is small as compared to the region $\left[X_{\mathrm{L}}, X\right]$ the occurrences of $1 \rightarrow 1$ are relatively rare in that case.

A part of a typical sequence for example is given by

$$
\ldots 1 r 1 r 1 r 1 r 1 r \underline{11} 1 \mathrm{r} 1 \mathrm{r} 1 \mathrm{r} 1 \mathrm{r} 1 \mathrm{r} 1 \mathrm{r} \operatorname{lr} 11 \mathrm{r} \operatorname{lr} 1 \mathrm{r} 11 \mathrm{r} 1 \mathrm{r} 1 \ldots
$$

When one considers an orbit of the twice iterated map $f^{2}(x)$ one follows either the even or the odd elements of the above sequence. That is, one sees

$$
\begin{aligned}
& \text { odd: } \quad \ldots 111111 \text { rrrerrrrr1111rr..., } \\
& \text { even: ...rrrrr111111111rrr111..., }
\end{aligned}
$$

which is intermittent band switching. Comparing the odd and even sequence we observe that a series of 1 's is always one element longer than the corresponding series of $r$ 's in the twin sequence.

Hence everything that applies to a phase of length $t$ in the right region applies to a phase of length $t+1$ in the left region. For instance for the minimal phase lengths discussed in the next section we have

$$
t_{\min , \mathrm{L}}=t_{\min , \mathrm{R}}+1
$$

where $t_{\min , \mathrm{L}}$ is the minimal phase length in the left region and $t_{\min , \mathrm{R}}$ is the minimal phase length in the right region. For the probabilities $P_{\mathrm{L}}(t)$ and $P_{\mathrm{R}}(t)$ for a phase of length $t$ to occur in the left, respectively, the right region we 
have in a similar fashion

$$
P_{\mathrm{L}}(t)=P_{\mathrm{R}}(t-1)
$$

So the switching process of an oribt of $f^{2}(x)$ amounts to an anomaly of the type 11 in the alternating sequence ... $\operatorname{lr} \operatorname{lr} 1 \mathrm{r} \ldots$ of an orbit of $f(x)$. Anomalies of the type 111 are not possible and neither are anomalies of the type $\mathrm{rr}$ reported in ref. [17]. The same considerations can be applied to the submaps of $f^{2^{n-1}}(x)$ at the band mergings at $a_{n}$, where the orbit of $f^{2^{n}}(x)$ switches between the regions $\left[\tilde{x}_{i}, x_{2^{n-1}, i}\right]$ and $\left[x_{2^{n-1}, i}, \tilde{x}_{2^{n}+i}\right], i=1,2, \ldots, 2^{n-1}$, where $x_{2^{n-1}, i}$ denotes the $i$ th element of the $2^{n-1}$-cycle.

\section{Minimal phase length}

Let us consider the region $x>X$ in fig. 10. An orbit of $f^{2}(x)$ in the right box will switch to the left region $x<X$ after it gets into the escape interval $I_{\text {esc, } \mathrm{R}}$. When this happens the orbit is transferred on the next iteration into the interval $\mathrm{I}_{0}=\left[X_{\mathrm{R}}, 1\right]$. The interval $\mathrm{I}_{\mathrm{c}}$ s mapped by $f^{2}(x)$ to $\mathrm{I}_{1}=\left[\tilde{x}_{3}, X\right]$, then to $I_{2}=\left[\tilde{x}_{5}, X\right]$, then to $I_{3}=\left[\tilde{x}_{7}, X\right]$. In fig. 10 the interval $I_{3}$ overlaps with the

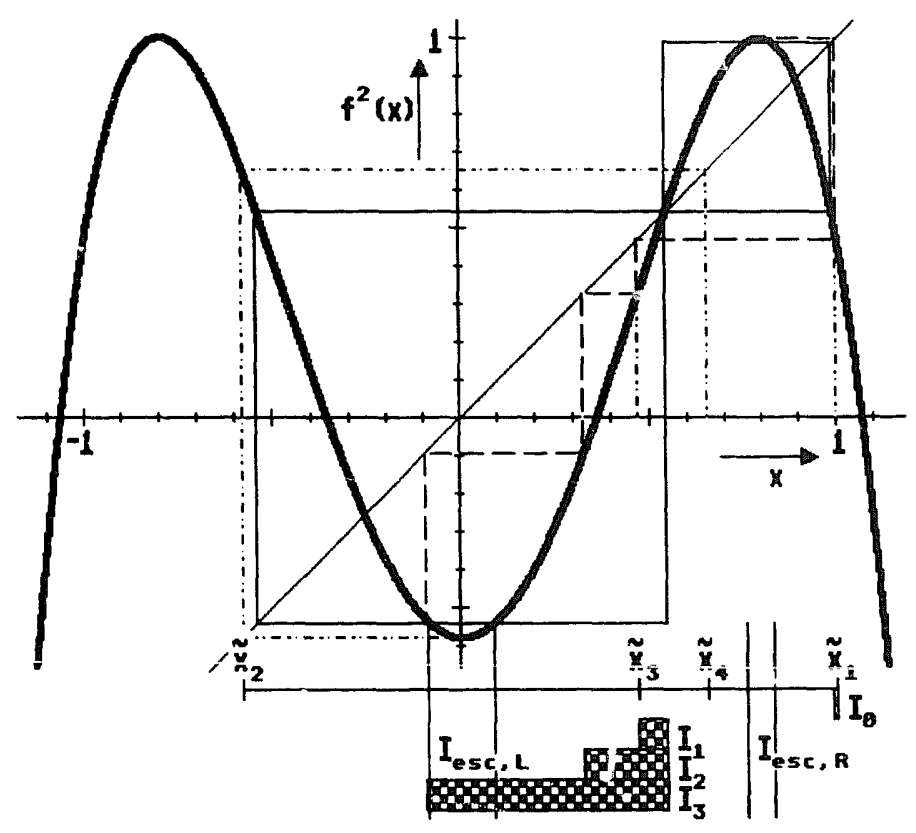

Fig. 10. The map $f^{2}(x)$ for $z=2$, at $a=1.58$, after the band merging. The boundzries of the band are $\tilde{x}_{1}=1$ and $\tilde{x}_{2}=1-a$. The escape interval $I_{\text {esc. } R}$ of the right region $:$ napped onto the interval $I_{0}$, and then successively onto $I_{1}, I_{2}$ and $I_{3}$. Note that the interval $I_{3}$ overlaps with the escape interval $I_{\text {esc. } .}$ of the left region. 
escape interval of the left box and thus the orbit has a possibility to switch back to the right region on the second next iteration (since the escape interval is first transferred to the interval $\left[\tilde{x}_{2}, X_{\mathrm{L}}\right]$ at the left). So we observe that in this case the orbit must spend at least four iterations of $f^{2}(x)$ in the left region. We define the minimal phase length in the left region $t_{\min , \mathrm{L}}$ as the minimal number of iterations spent in that region. In general $t_{\min , \mathrm{L}}$ will depend on the value of $\varepsilon=a-a_{1}$ as well as on the order $z$ of the maximum. In the above example we have $t_{\min , \mathrm{L}}=4$.

To establish an accurate lower bound for $t_{\min }$ as a function of $\varepsilon$ we determine the minimal number $n$ of iterations of $f^{2}(x)$ needed to map $\tilde{x}_{3}$ into the escape region $I_{\text {esc.L }}$ (or to the left of it). For small values of $\varepsilon$ the region $I_{1}$ is quite narrow, with $\tilde{x}_{3}$ close to the unstable fixed point $x$, and the number of iterations can be found from

$$
\tilde{x}_{3+2 n}-X=\left[f^{\prime}(X)\right]^{2 n}\left(\tilde{x}_{3}-X\right) .
$$

The escape interval lies in a small neighbourhood of the minimum at $\tilde{x}_{0}=0$ and as soon as $\tilde{x}_{3+2 n}$ reaches this point the orbit has a possibility to escape after one extra iteration. Hence from (12) we can estimate the minimal number of iterations $n_{\mathrm{L}}$ of $f^{2}(x)$ to get from $\tilde{x}_{3}$ to the escape region:

$$
n_{\mathrm{L}}=-\frac{1}{2} \ln \left(\frac{X-\tilde{x}_{3}}{X-\tilde{x}_{0}}\right) / \ln \left|f^{\prime}(X)\right|
$$

Since a non-integer number of iterations has no meaning, it is understood that $n_{\mathrm{L}}$ should be lifted to the next integer, i.e. $n_{\mathrm{L}} \rightarrow\left[n_{\mathrm{L}}+1\right]$. Here we may anticipate that for small $\varepsilon$ eq. (13) will give a very good estimate of the number of iterations $n_{\mathrm{L}}$, although of course the linear approximation (12) will not be good for values of $\tilde{x}_{3+2 n}$ close to $\tilde{x}_{0}$. This is due to the fact that such values are reached from points where the linear approximation is valid only by a small number of iterations.

The minimal phase length for an orbit of $f^{2}(x)$ in the left region $t_{\min , \mathrm{L}}$ is then $\left[n_{\mathrm{L}}+3\right]$, since one iteration is needed to get into the interval $I_{1}$ and another one is needed to escape to the interval $\left[\tilde{x}_{2}, X_{\mathrm{L}}\right]$ before switching to the right region.

For the right region the same arguments can be applied. However, the length of phases of $f^{2}(x)$ in the right region is always 1 iteration shorter than the length of the corresponding phases in the left region, as we showed in the previous section. In fact, we can read this directly from the analogue of eq. (13) for the right region, taking into account that $I_{\mathrm{es} r . \mathrm{R}}$ is located around the pre-image at the right of $\tilde{x}_{0}$ which we denote by $\tilde{x}_{-1}$. We thus have 


$$
n_{\mathrm{R}}=-\frac{1}{2} \ln \left(\frac{\tilde{x}_{4}-X}{\tilde{x}_{-1}-X}\right) / \ln \left|f^{\prime}(X)\right|
$$

Substitution of the approximate relations

$$
\begin{aligned}
& \tilde{x}_{4}-X \approx f^{\prime}(X)\left(\tilde{x}_{3}-X\right), \\
& \tilde{x}_{-1}-X \approx\left(\tilde{x}_{0}-X\right) / f^{\prime}(X)
\end{aligned}
$$

immediately yields

$$
n_{\mathrm{R}}=n_{\mathrm{L}}-1
$$

Thus the minimal phase lengths in the left and the right region are related by $t_{\min , \mathrm{L}}=t_{\min , \mathrm{R}}+1$. Consequently the minimal phase length $t_{\min }$ is equal to $t_{\min , \mathrm{R}}=\left[n_{\mathrm{R}}+3\right]=\left[n_{\mathrm{L}}+2\right]$.

From now on, we shall concentrate on the left region phenomena only, i.e. we shall only use the quantities $t_{\min , \mathrm{L}}, P_{\mathrm{L}}$, etc.

The quantities in eq. (13) can be calculated near the band merging using simple perturbation techniques. We shall do this for the map $(1), f(x)=$ $1-a|x|^{z}$.

We have

$$
\tilde{x}_{0}=0, \quad \tilde{x}_{1}=1, \quad \tilde{x}_{2}=1-a, \quad \ddot{x}_{3}=1-a|1-a|^{z},
$$

and so on.

Inserting $a-a_{1}+\varepsilon$ we obtain

$$
\tilde{x}_{3}(\varepsilon)=1-\left(a_{1}+\varepsilon\right)\left|1-\left(a_{1}+\varepsilon\right)\right|^{z}
$$

For the fixed point $X$ we observe that when the minimum in fig. 10 touches the bottom of the left box we have $X=X_{\mathrm{L}}=\tilde{x}_{2}$, hence $X=a_{1}-1$ at the band merging. The fixed point relation $f(X)=X$ thus yields

$$
\left(a_{1}-1\right)^{z}=\left(2-a_{1}\right)=\left(2-a_{1}\right) / a_{1} .
$$

Expanding (18) we obtain, using the above relation,

$$
\tilde{x}_{3}(\varepsilon)=a_{1}-1-\left(\frac{2-a_{1}}{a_{1}}\right)\left(\frac{(z+1) a_{1}-1}{a_{1}-1}\right) \varepsilon+\mathcal{O}\left(\varepsilon^{2}\right) .
$$

On the other hand, inserting $X=a_{1}-1+\phi(\varepsilon)$, with $\phi(0)=0$, into $f(X)=$ 
$X$ and expanding one finds

$$
X(\varepsilon)=a_{1}-1+\left(\frac{2-a_{1}}{a_{1}}\right)\left(\frac{a_{1}-1}{(z-1) a_{1}-2 z+1}\right) \varepsilon+\mathcal{O}\left(\varepsilon^{2}\right)
$$

So, summarizing, we find the following expressions:

$$
\begin{aligned}
& X-\tilde{x}_{3}=\left(\frac{z\left(2-a_{1}\right)}{a_{1}\left(a_{1}-1\right)}\right)\left(\frac{z a_{1}^{2}-2(z+1) a_{1}+2}{(z-1) a_{1}-2 z+1}\right) \varepsilon+\mathcal{O}\left(\varepsilon^{2}\right), \\
& X-\tilde{x}_{0}=a_{1}-1+\mathcal{O}(\varepsilon) \\
& f^{\prime}(X)=\frac{-z\left(2-a_{1}\right)}{a_{1}-1}+\mathcal{O}(\varepsilon) .
\end{aligned}
$$

For the case $z=2$ for example this yields

$$
t_{\operatorname{min.L}}=[3-0.965 \ln (3.43 \varepsilon)] \text {. }
$$

Applying eq. (25) we find the values $t_{\min , \mathrm{L}}=6,8,10,12,15$ for $\varepsilon=10^{-2}$, $10^{-3}, 10^{-4}, 10^{-5}, 10^{-6}$, respectively. These values are in perfect agreement with the numerical values obtained by iteration of the interval $\mathrm{I}_{1}=\left[\tilde{x}_{3}, X\right]$.

Some of the considerations given above are reminiscent of an estimate by Grebogi et al. [6] of the phase length of the transient chaotic phase in the case of intermittent bursting. The interpretation, however, is quite different and the laminar phase in intermittent bursting does not show a minimal phase length effect. Further, in ref. [7] some plots of distributions of phase lengths were shown, but the minimal phase length phenomenon was not mentioned.

\section{Probabilities of the short phase lengths}

In order to calculate the probabilities of the first occurring phasi lengths we follow the switching process in detail once again. An orbit of $f^{2}(x)$ that switches from right to left first gets into the right escape region. When the orbit is chaotic enough (i.e. when the value of the chaos-parameter $a$ is not inside or very close to a periodic window), and the escape region is small, it is reasonable to assume that the points of entrance into the escape region $I_{\text {esc, } R}$ are uriformly distributed over that region, that is

$$
\rho_{\text {esc }, \mathrm{R}}=\text { constant } .
$$


The escape interval $\mathrm{I}_{\text {esc, } \mathrm{R}}$ is mapped under $f^{2}(x)$ onto the interval $\mathrm{I}_{0}=$ $\left[X_{\mathrm{R}}, 1\right]$, cf. fig. 10. The (normalized) distribution $\rho_{0}(x)$ of points mapped on this interval is obtained by applying the Frobenius-Perron operator (see appendix B) to the (constant) distribution $\rho_{\mathrm{esc}, \mathrm{R}}^{\mathrm{R}}(x)$, that is

$$
\begin{aligned}
\rho_{0}(x) & =\int_{\mathbf{I}_{\mathrm{esc}, \mathrm{R}}} \rho_{\mathrm{esc}, \mathrm{R}} \delta\left(x-f^{2}\left(x^{\prime}\right)\right) \mathrm{d} x^{\prime} \\
& =z^{-1}\left(1-X_{\mathrm{R}}\right)^{-1 / z}(1-x)^{-1+1 / z}, \quad x \in \mathrm{I}_{0} .
\end{aligned}
$$

This distribution has a cusp type singularity at $x=1$ for all $z>1$. In the limit $z \downarrow 1$ it straightens out to a uniform distribution. On the next iteration $\mathrm{I}_{0}$ is mapped onto $\mathrm{I}_{1}$, with a distribution $\rho_{1}(x)$ having a cusp type singularity at $\tilde{x}_{3}$, i.e. at the left boundary of the interval $I_{1}$. We have

$$
\rho_{1}(x)=z^{-1}\left(X-\tilde{x}_{3}\right)^{-1 / z}\left(x-\tilde{x}_{3}\right)^{-1+1 / z}, \quad x \in \mathbf{I}_{1} .
$$

In order to find the probabilities for the shorter phases to occur we iterate the left escape region $\mathrm{I}_{\text {esc. } \mathrm{L}}$ backwards in time (under $f^{2}(x)$ ). The successive pre-images of $\mathbf{I}_{\text {esc. } \mathrm{L}}$ form a structure on the $x$-axis whose complement is a (multifractal) Cantor set, as illustrated in fig. 11.

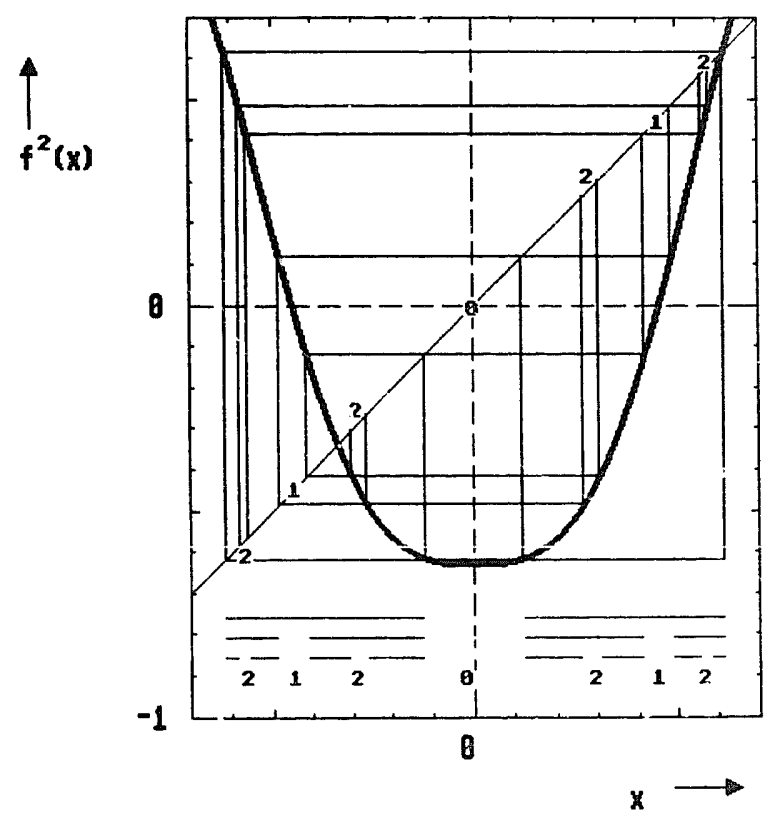

Fig. 11. The map $f^{2}(x)$ for $z=3$ at $a=1.63$. Only the part corresponding to the left box is plotted. Some pre-images under $f^{3}(x)$ of $\mathrm{I}_{\mathrm{esc}, \mathrm{L}}$ are depicted. They arc grouped in a tree structure along the $x$-axis. The complement is a multifractal Cantor set. 
An orbit of $f_{2}(x)$ that lands in one of the $2^{n} n$th pre-images of $I_{\text {esc, } \mathrm{L}}$ will switch to the right region after precisely $n+1$ iterations $\left(\right.$ of $f^{2}(x)$ ). The corresponding phase length in the left region is then $t_{\mathrm{L}}=n+2$ because to land left in the first place counts already for one iteration. The probability for a phase of length $t_{\mathrm{L}}$ in the left region to occur equals then

$$
P_{\mathrm{L}}(t)=\sum_{i=1}^{2^{t-2}} \int_{f_{(i)}^{-2(t-2)}\left(\mathrm{I}_{\mathrm{esc}, \mathrm{L}}\right)} \rho_{1}(x) \mathrm{d} x
$$

where $i$ labels the $(t-2)$ th pre-images of $\mathrm{I}_{\text {esc, } \mathrm{L}}$.

The overlap of an arbitrary interval $[a, b]$ in the left region with $\rho_{1}(x)$ can be simply calculated to be

$$
\begin{aligned}
\int_{a}^{b} \rho_{\mathrm{i}}(x) \mathrm{d} x & =\left[\left(\frac{x-\tilde{x}_{3}}{X-\tilde{x}_{3}}\right)^{1 / z}\right]_{\max \left(a, \tilde{x}_{3}\right)}^{\max \left(b, \tilde{x}_{3}\right)} \\
& =\theta\left(b-\tilde{x}_{3}\right)\left[\left(b-\tilde{x}_{3}\right)^{1 / z}-\theta\left(a-\tilde{x}_{3}\right)\left(a-\tilde{x}_{3}\right)^{1 / z}\right]\left(X-\tilde{x}_{3}\right)^{-1 / z},
\end{aligned}
$$

$\theta(x)$ being the step function. So, by iterating backward (under $f^{2}(x)$ ) the leif escape region, which is given by

$$
I_{\text {esc }, \mathrm{L}}=\left[-\left(\frac{1-\left(\frac{1+X}{a}\right)^{1 / z}}{a}\right)^{1 / z}, \quad\left(\frac{1-\left(\frac{1+X}{a}\right)^{1 / z}}{a}\right)^{1 / z}\right],
$$

and by applying eqs. (29) and (30) we obtain theoretically the probabilities for all possible phase lengths.

In practice this method can only be used for short phase lengths, since in order $t c$; wbiain the $P_{\mathrm{L}}(t)$ we must calculate $2^{t-2}$ pre-images of $\mathrm{I}_{\text {esc, } \mathrm{L}}$ and their corresonding overlaps. We have done this for several $z$ and $\varepsilon=a-a_{1}>0$ and for values of $\varepsilon$ not too close to a periodic window we find a good agreement between the predicted probabilities according to (29) and (30) and numerically obtained phase length probabilities. In fig. 12 we present the result of such a confrontation for $z=2$ and $a=1.55\left(a_{1}=1.543689 \ldots\right)$. The stars represent the theoretical values, and the bars represent the numericaliy measured values, $\operatorname{tog}$ sther with their error estimates. The latter are given oy

$$
\Delta p=\sqrt{\frac{p(1-p)}{N}},
$$

where $p$ is $P_{\mathrm{L}}(t)$ and $N$ the total number of measured phases. 


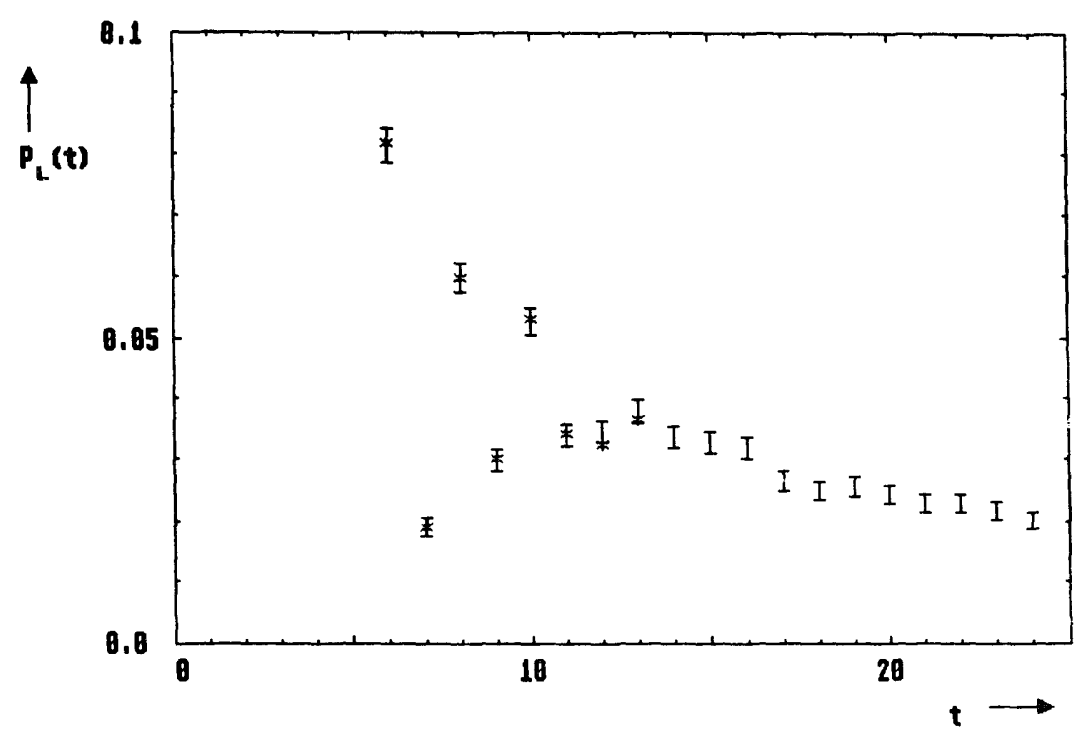

Fig. 12. Comparison between numerically obtained phase length probabilties (bars) for $z=2$ and $a=1.55$, and their theoretically predicted values (stars) (see text). Here $t_{\min . \mathrm{L}}=6$, i.e. $P_{\mathrm{L}}(t)=0$ for $t<6$.

Clearly the first pre-image of $I_{\text {esc. } \mathrm{L}}$ that starts having an overlap with $\rho_{1}(x)$ is the $\left(t_{\min . \mathrm{L}}-2\right)$ th pre-image of $I_{\text {esc.L }}$ that is the closest to the fixed point $X$. We expect that the probability of this shortest phase length $P_{\text {max.L }}\left(t_{\min . \mathrm{L}}\right)$ is maximal when $\rho_{1}(x)$ has the greatest overlap with the relevant pre-image of $I_{\text {esc.L. }}$, i.e. when

$$
\tilde{x}_{3}=\max _{i} f_{(i)}^{-2\left(t_{\min . \mathrm{L}}-2\right)}\left(\tilde{x}_{0}-\frac{1}{2} L\right),
$$

where $L$ is the width of the left escape region.

The width of the successive right-hand side pre-images of $\tilde{I}_{\mathrm{esc}, \mathrm{L}}$ reduces approximately with a factor $\left[f^{\prime}(X)\right]^{2}$ each step, so the width of the relevant pre-image is $L\left[f^{\prime}(X)\right]^{-2\left(t_{\min . \mathrm{L}}-2\right)}$. Hence the maximal probability for the minimal phase length (in the left region) is estimated to be

$$
\begin{aligned}
P_{\max , \mathrm{L}}\left(t_{\min , \mathrm{L}}\right) & =\int_{\tilde{x}_{3}}^{\tilde{x}_{3}+L\left[f^{\prime}(X)\right]^{-2\left(t_{\min . \mathrm{L}}-2\right)}} z^{-1}\left(X-\tilde{x}_{3}\right)^{-1 / z}\left(x-\tilde{x}_{3}\right)^{-1+1 / z} \mathrm{~d} x \\
& =\left[\frac{L}{\left(X-\tilde{x}_{3}\right)\left[f^{\prime}(X)\right]^{2\left(t_{\min , L^{-2}}\right)}}\right]^{1 / z} \\
& =\left(\frac{L}{X+\frac{1}{2} L}\right)^{1 / z} \sim \varepsilon^{1 / z^{2}} .
\end{aligned}
$$

In the last steps we have used eq. (12) and the fact that $L \sim \varepsilon^{1 / z}$. 


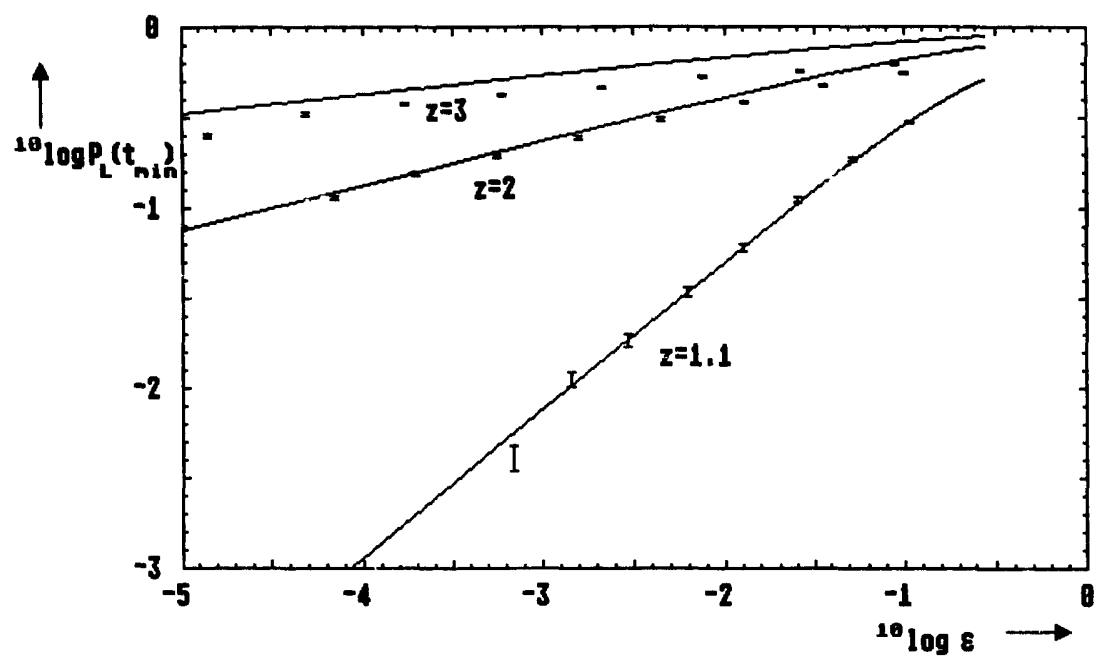

Fig. 13. Numerically obtained (logarithmic) probabilities of $t_{\min . \mathrm{L}}$ for $z=1.1, z=2$ and $z=3$, for values of $\varepsilon$ where a maximal value of $P_{\mathrm{L}}\left(t_{\min , \mathrm{L}}\right)$ is expected. The measured values are represented by bars, and they are compared to the estimates based on eq. (34), represented by the lines with slope $1 / z^{2}$.

In fig. 13 we compare the numerically measured (logarithmic) probabilities of $t_{\min , \mathrm{L}}$ for $z=1.1, z=2$ and $z=3$, for values of $\varepsilon$ where a maximal value of $P_{\mathrm{L}}\left(t_{\text {min, } \mathrm{L}}\right)$ is expected (bars), with their theoretical prediction according to eq. (34), represented by the lines with slope $1 / z^{2}$.

The maximal height of the peak as compared to the inverse of the average phase length grows with increasing $z$, since its scaling is governed by a smaller power of $\varepsilon$. In other words, the ennancemient of the shortest phase length increases rapidly for growing $z$. For actual systems such a feature may be used to gain information on the $z$-value of the underlying map.

We ouserve that the agreement between theory and numerical mesurement is quite good, although for higher values of $z$ the results start diverging. This is due to the fact that PM and IB intermittency effects near periodic windows are much more persistent for higher values of $z$. Also the actual size of the periodic windows increases for growing $z$. These features are discussed in the next section.

\section{The influence of windows}

Finally, for $\varepsilon$-values in the neighbourhood of windows different probability distributions are found $[17,23,25,26]$, since an intricate interplay between various types of intermittency occurs here. For small values of $\varepsilon=a-a_{1}$ almost all windows have odd $(2 n-1)$ period, and they are ordered in a tree 
structure along the $z$-axis. For $\varepsilon$-values inside these wincows we only have the phase length $t_{\mathrm{L}}=n\left(t_{\mathrm{R}}=n-1\right)$. In order to see how this comes about we consider again the map $f^{2}(x)$ in fig. 11 , for $z=3$ and $a=1.63$. The left escape interval $I_{e s c, L}$ is centered around the critical point $\tilde{x}_{0}$, and consequently all pre-images under $f^{2}(x)$ of $\mathrm{I}_{\text {esc, } \mathrm{L}}$ are centered around the pre-images of $\tilde{x}_{0}$; the latter are thus ordered in the same (tree)- structure as the former.

Now, when $a$ is increased from $a_{1}$ to $a_{0}=2$, or $\varepsilon$ from 0 to $2-a_{1}$, the value of $\tilde{x}_{3}=f^{3}\left(\tilde{x}_{0}\right)$ moves leftward monotonically from the fixed point $X$ to the left attractor boundary $\tilde{x}_{2}$. Each time $\tilde{x}_{3}$ passes a pre-image $f^{-2 i}\left(\tilde{x}_{0}\right)$ we have a superstable period $m=2 i+3$-cycle, characterizing a window of the same period around it. From this it is inferred that there is a family of odd windows which is organized in a tree structure along the $a$-axis, with $a=a_{1}$ as an accumulation point.

The widths of the windows in this family can be calculated explicitly [24, 27], and for small $\varepsilon$ an accurate scaling for the width as well as the position can be estimated. In fig. 14 below we have given a plot of the log of the mean phase length ${ }^{10} \log \left\langle t_{\mathrm{L}}\right\rangle$ as a function of ${ }^{10} \log \varepsilon$, for $z=3$. The plateaus correspond to the periodic windows, and the tree structure is clearly discernible. This plot is reminiscent of fig. 3 in ref. [17], but there only part of the odd windows were discussed.

Just before the opening of a perodic window we observe PM iniermittency and just after the closing intermittent bursting (IB) takes place. Thesf, intermittencies occur simultaneously with the switching process. In fig. 15 a time series is depicted of the map $f^{2}(x)$, for $z=2$ and $a=1.57471$, just befo:e the opening of the first period-7 window at $a=1.57471570$. . From this picture the interplay between PM intermittency and intermittent switching is evident. As one observes we still have intermittent switching during the laminar phases of the PM intermittency. Indeed, during the latter we alternatingly have 4

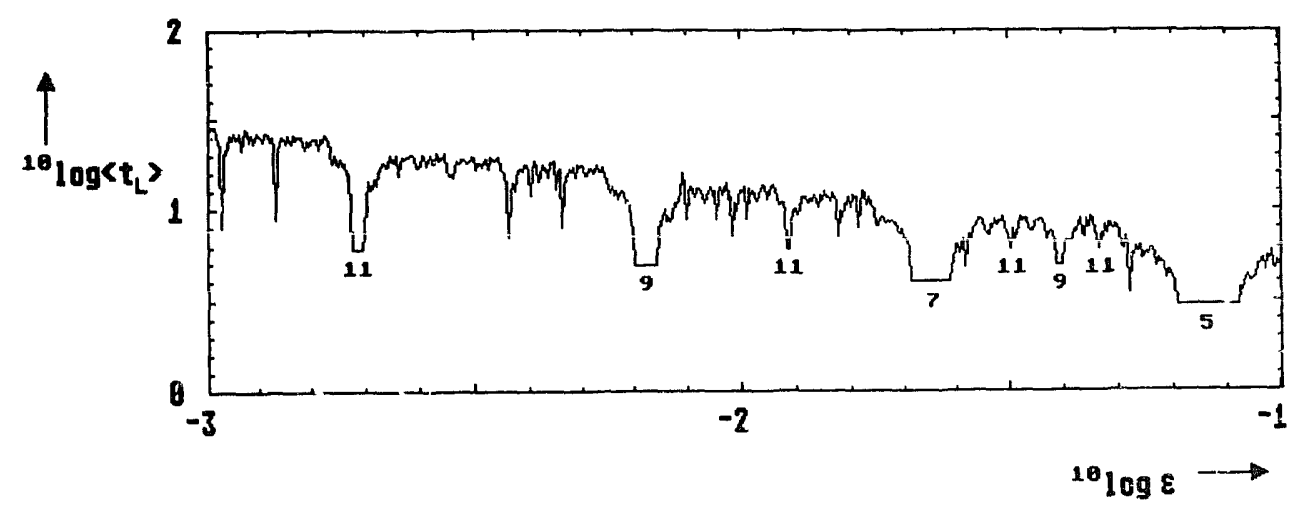

Fig. 14. ${ }^{10} \log \left\langle t_{\mathrm{L}}\right\rangle$ versus ${ }^{10} \log \varepsilon$, for $z=3$. Within a period $2 n-1$ window we have $\left\langle t_{\mathrm{L}}\right\rangle=n$. 


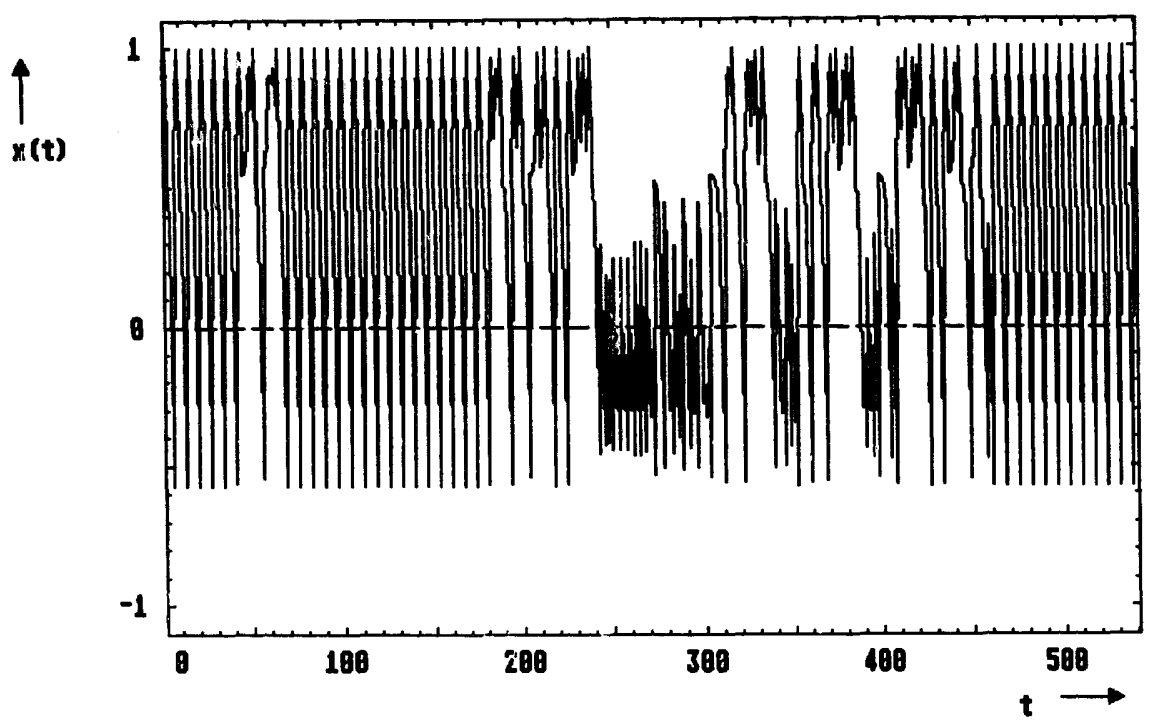

Fig. 15. A time series of the map $f^{2}(x), f(x)=1-a|x|^{2}$, for $a=1.57471$, just before the opening of the 7-window at $a=1.57471570 \ldots$ One can easily discern Pomeau-Manneville type 1 intermittency (e.g. between $t=70$ and $t=180$ ) and intermixtent switching (e.g. between $t=230$ and $t=430$ ). Successive iterates are joined by straight lines for clarity.

consecutive orbit points of $f^{2}(x)$ in the left region and after that 3 in the right region.

Our probability analysis for the phase lengths does not hold anymore in cases like the above, because the requirement that the points of entrance in the escape intervals are uniformly distributed is violated here. However, if we consider all phase lengths other than 4 (in the left rugion), and normalize the remaining theoretically and numerically obtained distributions in the proper way, we still have a good agreement, as can be checked in fig. 16 where we have compared theory and numerics for the above parameter values discarding $t_{\mathrm{L}}=4$.

Finally, in fig. 17, a discrete Fourier spectrum is given of a time series of $N=2^{17}$ iterations of the single map $f(x)$, still for $z=2$ and $a=1.57471$. We can clearly distinguish the peaks in the spectrum for period 7 and period 2 . In the figure we have plotted the $\log$ of $S_{k}=\left|C_{k}\right|^{2}$, with

$$
r-\stackrel{N-1}{\Gamma}-2 \pi i k t
$$


T. Post et al. / Phase lengths in intermittent band switching

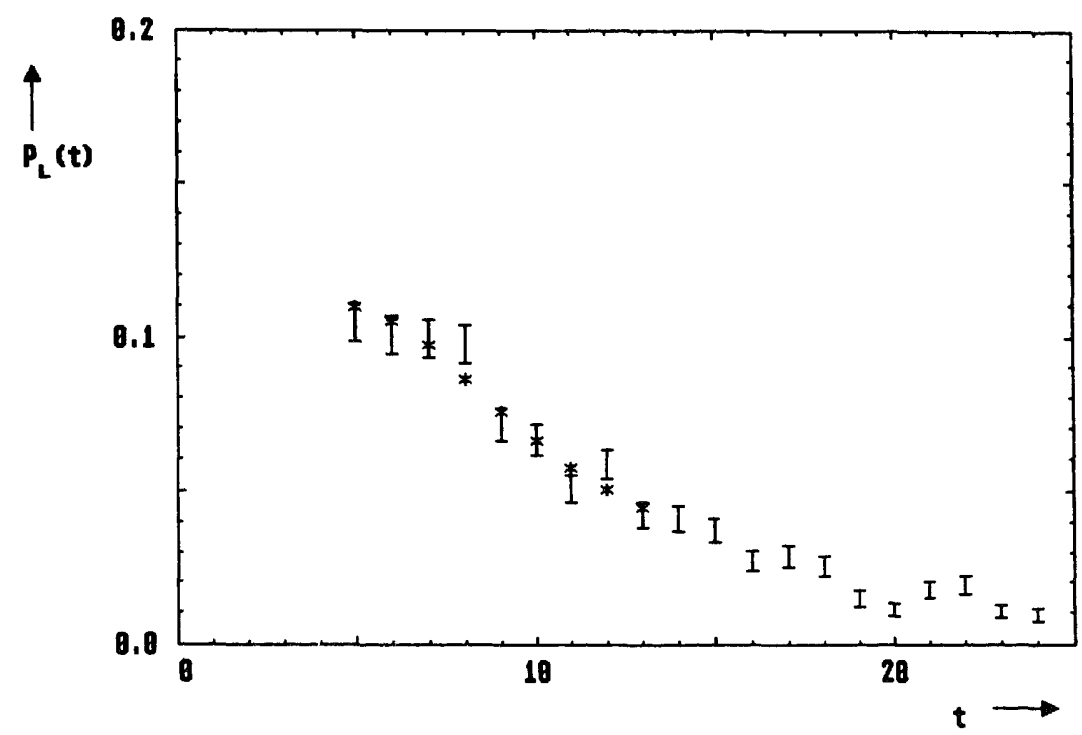

Fig. 16. Comparison between numerically obtained phase length probabilities (bars) for $z=2$ and $a=1.57471$, and their theoretically predicted values (stars). The minimal length $t=4$ is left out of consideration (see text).

period $2^{n}$ spectral peaks as a function of $\varepsilon=a-a_{n}$ is discussed in refs. $[5,17,28]$; they obey the same scaling law as the reciprocal mean phase length, namely $\Delta k \sim \varepsilon^{1 / z}$.

In refs. $[24,27]$ it is shown that the portion of parameter space that is covered by the periodic windows is a monotonically increasing function of $z$.

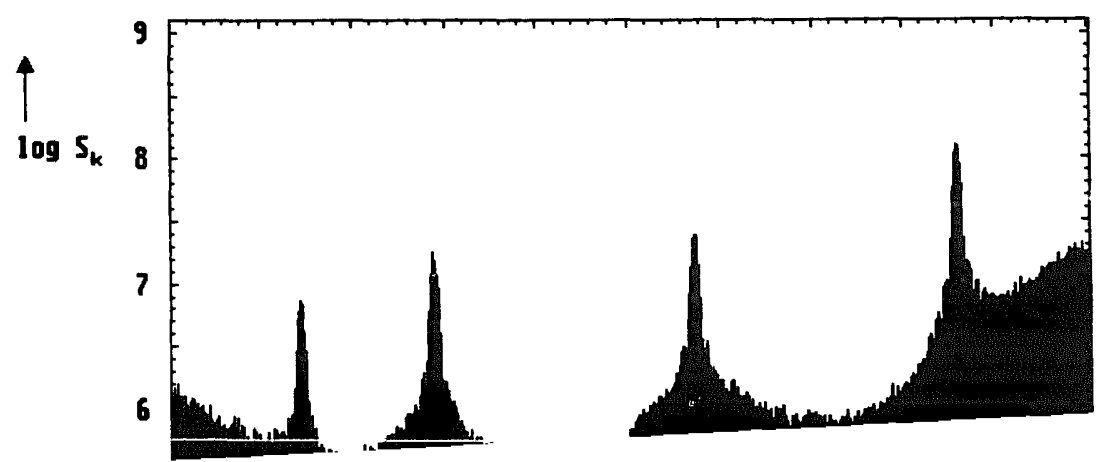


The PM intermittency, and especially the intermittent bursting behaviour that are associated with the windows also cover a larger part of parameter space for larger values of $z$.

\section{Appendix A}

The position of the $2^{k}$ chaotic bands between $a_{k+1}$ and $a_{k}$

In this appendix we discuss a simple recipe to obtain the exact position of the chaotic bands in the band merging cascade.

Firstly, we observe that the eventual $2^{k}$ chaos bands are bounded by the values of the extrema of $f^{2^{k}}(x)$ and their images under the mentioned composite function. The values of the extrema are just the $\tilde{x}_{i}, i=1, \ldots, 2^{k}$ (with $\tilde{x}_{i}=f^{i}\left(\tilde{x}_{0}\right), \tilde{x}_{0}$ being the single critical point $\left(f^{\prime}\left(\tilde{x}_{0}\right)=0\right)$ of the unimodal mapping).

Proof. For an extremum of $y=f^{n}(x)$ we have $\mathrm{d} f^{n}(x) / \mathrm{d} x=0$. Applying the chain rule this reads

$$
f^{\prime}\left(f^{n-1}(x)\right) f^{\prime}\left(f^{n-2}(x)\right) \cdots f^{\prime}(f(x)) f^{\prime}(x)=0,
$$

so we have

$$
f^{n-1}(x)=\tilde{x}_{0} \vee f^{n-2}(x)=\tilde{x}_{0} \vee \cdots \vee f(x)=\tilde{x}_{0} \vee x=\tilde{x}_{0},
$$

and hence the value $y=f^{n}(x)$ of an extremum is given by

$$
y=\tilde{x}_{1} \vee y=\tilde{x}_{2} \vee \cdots \vee y=\tilde{x}_{n-1} \vee y=\tilde{x}_{n} .
$$

Thus the boundaries of either of the $2^{k}$ chaos bands are $\tilde{x}_{i}$ and $\tilde{x}_{2^{k}+i}$, $i=1, \ldots, 2^{k}$. Their ordering follows from the observation that the band merging cascade is the "reverse" of the period-doubling process. However imperfect this mirror-image may be, a sure thing is that for $a<a_{k}$ (the value where $2^{k}$ bands merge into $2^{k-1}$ ) the quantities $\tilde{x}_{0}, \ldots, x_{2 k+1}$ have the same order as the corresponding elements of a superstable $2^{k+1}$-cycle in the perioddoubling tree.

The order of superstable cycle elements follows from the theory of Metropolis, Stein and Stein (MSS) [22]. According to this theory any superstable $n$-cycle is characterized by a pattern of $n-1$ binary symbols. The first element of the cycle (numbered 0 or $n$ ) is always the critical point $\tilde{x}_{0}$ of the map and is 
not explicitly written in the pattern. The subsequent $n-1$ elements are represented by an $\mathrm{L}$ or an $\mathrm{R}$ according to their position being to the left or to the right of the critical point.

Now assume the map has a maximum. Then the superstable 2-cycle is represented by a single R. According to MSS the period-doubled version of any pattern $\mathrm{P}$ (called the harmonic $\mathrm{H}$ or $\mathrm{P}$ ) is obtained by applying the following rule:

$$
\begin{array}{ll}
\text { if the number of } R \text { 's in } P \text { is odd } & H=P L P, \\
\text { if the number of } R \text { 's in } P \text { is even } & H=P R P .
\end{array}
$$

So the pattern of any superstable $2^{k}$-cycle is obtained by repeatedly applying the above rule to the 2-cycle:

$$
\mathrm{R} \rightarrow \mathrm{RLR} \rightarrow \text { RLRRRLR } \rightarrow \text { RLRRRLRLRLRRRL.R } \rightarrow \ldots
$$

Since the cycle elements in the period-doubling tree cannot cross each other as $a$ is increased the order of the original cycle elements is preserved at each subsequent bifurcation (as also reflected by the rule (39)). At the $k$ th bifurcation at each cycle element labeled $i$ another one labeled $i+2^{k}$ is added immediately to the left or to the right of it. For the clement labeled 0 or $n$ the choice left/right is dictated by the letter in the rule (39).

Now, considering the mapping we obse ve the following:

- applying the map on two points to the left of $\tilde{x}_{0}$ preserves the order of the points;

- applying the map on two points to the right of $\tilde{x}_{0}$ reverses the order of the points.

Furthermore, considering the rule (39) we observe that a cycle point and its twin, half a cycle further, are always on the same side of $\tilde{x}_{0}$ (except $\tilde{x}_{0}$ itself of course).

So, reading the pattern we can construct the complete order of the cycle elements as follows:

Suppose we know the order of the $2^{k}$-cycle. Then from the pattern of the $2^{k+1}$-cycle we read the order of the elements $0\left(2^{k+1}\right)$ and $2^{k}$. If the $2^{k}$ th element of the pattern is $\mathrm{R}$ we have $\tilde{x}_{2^{k}}>\tilde{x}_{0}$, otherwise $\tilde{x}_{2^{k}}<\tilde{x}_{0}$. In either case, since $\tilde{x}_{0}$ is a maximum, we have $\tilde{x}_{2^{k}+1}<\tilde{x}_{1}$. Next, observing that the first letter of any pattern is $\mathbf{R}$, the subsequent pair has its order flipped: $\tilde{x}_{2^{k}+2}>\tilde{x}_{2}$. The second letter of any pattern is always $L$, so the next pair has the same order as the previous one: $\tilde{x}_{2^{k}+3}>\tilde{x}_{3}$. Reading the whole pattern we reconstruct the complete order of the cycle elements.

However, watching the rule (39) again, we can simplify this procedure 


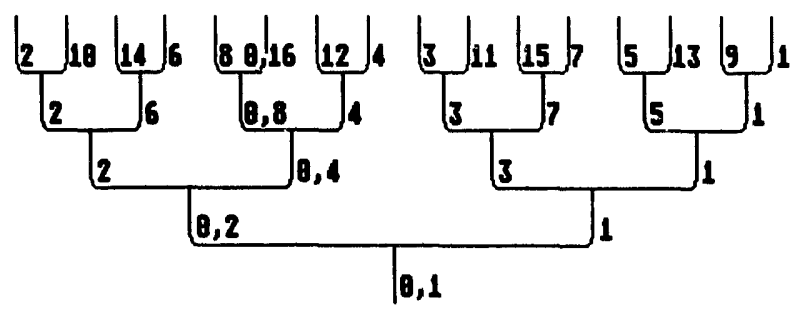

RLRRRLR L RLRRRLR

RLR R RLR

R L R

R

Fig. 18. Schematic representation of a part of the bifurcation tree of a unimodal map with a maximum. The ordering of the cycle elements as well as the corresponding pattern is indicated.

considerably. Firstly, we observe that that the added pattern element (number $2^{k}$ ) in the middle of the harmonic alternates from bifurcation to bifurcation. Secondly, as remarked above, we always have $\tilde{x}_{2^{k}+1}>\tilde{x}_{1}$. Thirdly, up to the $2^{k}$ th element the pattern equals the one of the $2^{k}$-cycle, and from this we infer that the order between $\tilde{x}_{i}$ and $\tilde{x}_{2^{k}+1}$ is the same as between $\tilde{x}_{i}$ and $\tilde{x}_{2^{k-1}+i}$.

Hence for the construction of the bifurcation tree we obtain the following simple recipe:

- In going from a 1-cycle to a 2-cycle the element labeled 1 forks to the right. (And hence the element labeled 0 or 2 to the left.)

- Cnce an element forks in a certain direction it makes the same choice at all subsequent bifurcations. This goes for all elements except for the one labeled 0 which forks in alternating directions. However, if we label this element $2^{k}$ instead of 0 , it follows the recipe perfectly.

A labeled bifurcation tree is drawn in fig. 18 .

When $\tilde{x}_{0}$ is a minimum the recipe is the same, only the first bifurcation has the extra element added to the left instead of to the right.

\section{Appendix B}

\section{The Frobenius-Perron operator}

In this appendix we show how distributions change when a mapping is applied on them (see alsc e.g. refs. $[29,30]$ ).

Suppose we have a probability distribution $\rho(x)$ of poinis on the real axis. We then ask ourselves what the probability distribution $\rho^{\prime}(x)$ looks like when we apply a map $f(x)$ on these points. Let us consider a plot of a map $f$ as in fig. 19.

The probability of finding a mapped point in some infinitesimal interval $\mathrm{d} x$ is given by $\rho^{\prime}(x) \mathrm{d} x$. This quantity must be equal to the probability of finding an original point in one of the pre-images under $f(x)$ of $\mathrm{d} x$. The width of a 


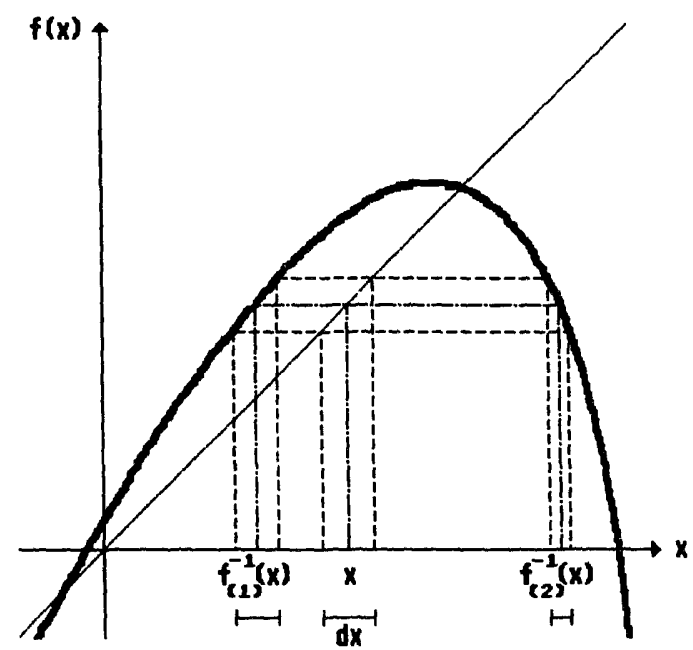

Fig. 19. Plot of an arbitrary map $f(x)$. In this case the points in the small interval $\mathrm{d} x$ come from 2 pre-images of $\mathrm{d} x$ under $f(x)$.

pre-image of $\mathrm{d} x$ is $\mathrm{d} x /\left|f^{\prime}\left(f^{-1}(x)\right)\right|$, as can also be verified from fig. 19. This continuity argument thus yields

$$
\rho^{\prime}(x) \mathrm{d} x=\sum_{(i)} \rho\left(f_{(i)}^{-1}(x)\right) \frac{\mathrm{d} x}{\left|f^{\prime}\left(f^{-1}(x)\right)\right|},
$$

where (i) labels the pre-images under $f$ of $x$. Eq. (41) can also be written as

$$
\rho^{\prime}(x)=\sum_{(i)} \int \frac{\rho\left(x^{\prime}\right)}{\left|f^{\prime}\left(f_{(i)}^{-1}(x)\right)\right|} \delta\left(x^{\prime}-f_{(i)}^{-1}(x)\right) \mathrm{d} x^{\prime} .
$$

A well-known rule about delta functions states that if $a_{i}$ are all zeroes of a function $g(x)$ then

$$
\delta(g(x)) \equiv \sum_{i} \frac{\delta\left(x-a_{i}\right)}{\left|g^{\prime}\left(a_{i}\right)\right|}
$$

Applying this rule to the previous expression we finally have

$$
\rho^{\prime}(x)=\int \rho\left(x^{\prime}\right) \delta\left(f\left(x^{\prime}\right)-x\right) \mathrm{d} x^{\prime}=P_{f} \circ \rho(x) .
$$

$P_{f}$ is the so-called Frobenius-Perron operator. 


\section{References}

[1] D.J. Tritton, Physical Fluid Dynamics (Van Nostrand/Reinhold, Wokingham, 1977), pp. 16, 209, 235, 265, 291.

[2] P. Collet and J.-P. Eckmann, Iterated Maps on the Interval as Dynamical Systems, Progress in Physics, vol. 1 (Birkhäuser, Boston, 1980).

[3]P. Cvitanović, ed., Universality in Chaos, a reprint selection (Hilger, Bristol, 1984).

[4] Y. Pomeau and P. Manneville, Comm. Math. Phys. 74 (1980) 189, also reprinted in ref. [3].

[5] S.J. Shenker and L.P. Kadanoff, J. Phys. A 14 (1981) L23.

[6] C. Grebogi, E. Ott and J.A. Yorke, Physica D 7 (1983) 181.

[7] H. Fujisaka, H. Kamifukumoto and M. Inoue, Prog. Theor. Phys. Lett. 69 (1983) 333.

[8] C. Grebogi, E. Ott and J.A. Yorke, Phys. Rev. Lett. 57 (1986) 1284.

[9] C. Grebogi, E. Ott, F. Romeiras and J.A. Yorke, Phys. Rev. A 36 (1987) 5365.

[10] C. Chen, G. Györgyi and G. Schmidt, Phys. Rev. A 35 (1987) 2660.

[11] M. Kitano, T. Yabuzaki and T. Ogawa, Phys. Rev. A 29 (1984) 1288.

[12] O. Sporns, S. Roth and F.F. Seelig, Physica D 26 (1987) 215.

[13] D. D.'Humières, M.R. Beasley, B.A. Huberman and A. Libchaber, Phys. Rev. A 26 (1982) 3483.

[14] R.W. Rollins and E.R. Hunt, Phys. Rev. A 29 (1984) 3327.

[15] H. Ishii, H. Fujisaka and M. Inoue, Phys. Lett. A 116 (1986) 257.

[16] R.M. Everson, Phys. Lett. A 122 (1987) 471.

[17] R. Brown, C. Grebogi and E. Ott, Phys. Rev. A 34 (1986) 2248.

[18] J.P. van der Weele, H.W. Capel and R. Kluiving, Physica A 145 (1987) 425.

[19] J.E. Hirsch, M. Nauenberg and D.J. Scalapino, Phys. Lett. A 87 (1982) 391, also reprinted in ref. [3].

[20] B. Hu and J. Rudnick, Phys. Rev. Lett. 48 (1982) 1645.

[21] M.J. Feigenbaum, J. Stat. Phys. 21 (1979) 669, also reprinted in ref. [3].

[22] N. Metropolis, M.L. Stein and P.R. Stein, J. Comb. Th. 15 (1973) 25, also reprinted in ref. [3].

[23] T. Post, H.W. Capel and J.P. van der Weele, Phys. Lett. A 133 (1988) 373.

[24] T Post, H.W. Capel and J.P. van der Weele, Phys. Lett. A 136 (1989) 109.

[25, T. Post, H.W. Capel and J.P. van der Weele, Proc. Conf. Nonlinear Dynamics, Bologna 1988, G. Turchetti, ed. (World Scientific, Singapore, 1989).

[26] T. Post, H.W. Capel and J.P. van der Weele, Proc. Conf. Singular Behaviour and Nonlinear Dynamics, Samos 1988, S. Pneumatikos, ed. (World Scientific, Singajore, 1989).

[27] T. Post, H.W. Capel and J.P. van der Weele, in preparation.

[28] F. Romeiras, C. Grebogi and E. Ott, Phys. Rev. A 38 (1988) 463.

[29] S.M. Ulam, Intersc. Tracts Pure \& Appl. Math. 8 (1960) 73.

[30] S. Grossmann and S. Thomae, Z. Naturforsch. 32a (1977) 1353. 